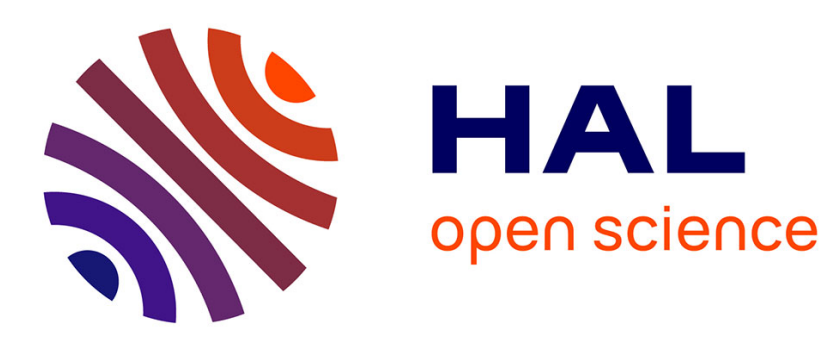

\title{
Homogenized Model with Memory for Two-Phase Compressible Flow in Double-Porosity Media
}

\author{
Mikhail Panfilov
}

\section{To cite this version:}

Mikhail Panfilov. Homogenized Model with Memory for Two-Phase Compressible Flow in DoublePorosity Media. Physics of Fluids, 2019, 31 (9), pp.093105. 10.1063/1.5120492 . hal-03035207

\section{HAL Id: hal-03035207 \\ https://hal.sorbonne-universite.fr/hal-03035207}

Submitted on 2 Dec 2020

HAL is a multi-disciplinary open access archive for the deposit and dissemination of scientific research documents, whether they are published or not. The documents may come from teaching and research institutions in France or abroad, or from public or private research centers.
L'archive ouverte pluridisciplinaire HAL, est destinée au dépôt et à la diffusion de documents scientifiques de niveau recherche, publiés ou non, émanant des établissements d'enseignement et de recherche français ou étrangers, des laboratoires publics ou privés. 


\title{
Homogenized Model with Memory for Two-Phase Compressible Flow in Double-Porosity Media
}

\author{
Mikhail Panfilov'1,2, a) \\ ${ }^{1)}$ Institut Elie Cartan, CNRS - Université de Lorraine, F-54506, Nancy, France \\ ${ }^{2)}$ Institut Jean le Rond d'Alembert, CNRS - Sorbonne Université, F-75252, Paris, \\ France
}

(Dated: 22 August 2019)

A completely averaged model of two-phase flow of compressible fluids in a medium with double porosity is developed. The variational asymptotic two-scale averaging method with splitting the nonlocality and nonlinearity is presented. Several mechanisms of delay are detected, as the nonequilibrium capillary redistribution of phases, pressure field relaxation caused by the compressibility, and the cross effects of fluid extrusion from pores due to the rock com- paction and fluid expansion. A generalized non-equilibrium capillary equation is obtained. All characteristic times of delay are explicitly defined as functions of saturation.

\section{INTRODUCTION}

A double porosity medium, which is also called ${ }^{53}$ fractured-porous medium, is the classical model applied ${ }^{54}$ to study the effects of memory or delay occurrence in ${ }^{55}$ fluid flow after changing the scale of examination. Such 56 a medium consists of low permeable blocks and highly ${ }^{57}$ permeable fractures. A single-phase compressible flow in 58 such a medium is characterized by the delay in pressure 59 behaviour. Indeed, in tight blocks, non-stationary pro- 60 cesses of perturbation propagation are much slower than ${ }^{6}$ in fractures, therefore, the average pressure in blocks re- 62 acts to a perturbation coming from fractures with a delay. ${ }^{6}$ As the result, the average pressure in the blocks depends 64 on the all history of variation of the average pressure in 65 the fractures, but not only on its current value, which 66 is the phenomenon of memory. Such a phenomenon is 67 observed in terms of the averaged pressures, i.e. on the 68 macroscopic scale. In the single-phase case, it is possi- 69 ble to obtain completely averaged model and to describe 70 explicitly the kernel of the memory operator. The first 71 mathematical model was suggested in ${ }^{11}$, where the con- 72 cept of a delay between the pressures was introduced. 73 Later, in ${ }^{9}$ and $^{22}$ it was shown by homogenization that ${ }_{74}$ high contrast in the permeability of blocks and fractures 75 leads to a long memory and changes the original diffusion 76 equation into an integro-differential homogenized equa- 77 tion. In some papers a more complete version of com- 78 pressible single-phase flow in double-porosity medium 79 was homogenized, by introducing elastic deformations of ${ }_{80}$ the medium described by Biot's equations, ${ }^{1},{ }^{21}$.

In the case of two-phase flow, another type of mem- 82 ory arises, which is caused by the capillarity. The cap- ${ }_{83}$ illary forces push the more wetting fluid from fractures ${ }_{84}$ into blocks, while displacing the non-wetting fluid from ${ }_{85}$ the blocks to the fractures. This phenomenon is known ${ }_{86}$ as the counter-current spontaneous imbibition. In par- ${ }_{87}$ ticular, this mechanism is essential for oil recovery by 88

\footnotetext{
a) mikhail.panfilov@univ-lorraine.fr
}

water injection in heterogeneous reservoirs, ${ }^{20}$. With respect to the flow in fractures, the spontaneous imbibition in blocks is very slow, which causes the capillary delay effects in terms of the averaged saturation of water (the term "saturation" means the volume fraction).

The capillary imbibition can be represented in terms of the propagation of the wave of water saturation from the block boundary towards the block centre. The dynamics of this wave is nonlinear, proper to two-phase flow in general. In classical two-phase model the counter-current imbibition is described by a nonlinear diffusion equation with nonlinear boundary conditions. We are, thus, faced with a situation where delay/memory (or time nonlocality) at the macroscale is caused by the propagation of a nonlinear wave at the local scale.

In the flow is two-phase and compressible simultaneously, the two types of the memory mentioned above, caused by the capillarity and the compressibility, should interact in some way, which could generate new physical cross phenomena. The detection of such phenomena is the main objective of the present paper. For this, it was necessary to develop the macroscale model of two-phase compressible flow in double-porosity media.

Attempts to develop the model of two-phase flow in double porosity media have been undertaken essentially for incompressible fluids ${ }^{4},{ }^{7},{ }^{8},{ }^{10},{ }^{12},{ }^{13},{ }^{19},{ }^{22},{ }^{28}$. The appearance of nonlocality in a nonlinear system, mentioned above, is a strong obstacle for obtaining averaged model. This is why the problem remains open for two-phase case, even non-compressible. Two papers devoted to compressible two-phase flow in double-porosity media, ${ }^{2}, 8$, in which one phase was an ideal gas while the second one was incompressible, have illustrated even deeper problems of interference between the nonlocality and twice nonlinearity. The attempts to overcome this difficulty by linearizing the equations of capillary imbibition, as, for instance, in $^{6}, 7,{ }^{10}$, mean physically that the flow in blocks becomes single-phase.

As a result of such an imposition of nonlocality and nonlinearity, the models obtained are not completely averaged. Namely, macroscopic equations and the cell problems make up a coupled system of equations, which 
should be solved together. This means that macroscopic 151 equations are not spared from microscopic variables, and 152 cell problems are not spared from macroscopic variables.153 Despite the importance of such works for mathematics, 154 the significance of such models for physics is disputable.155 This is one of the main reasons why the results of the ho- 156 mogenization theory were not well received by the phys-157 ical community.

This is why, the physics of two-phase flow in fractured-159 porous media continue to be studied mainly experimen-160 tally, or numerically. Among the recent experiments, it is161 important to cite the studies based on transparent glass162 micromodels: ${ }^{17},{ }^{18},{ }^{25}$, and on real rock samples scanned163 by nuclear magnetic resonance technique: ${ }^{20}$. The numer-164 ical modeling of the first kind is based on Darcy's scale165 model: ${ }^{14},{ }^{15},{ }^{23},{ }^{25},{ }^{26}$, in which the main problem is fo-160 cused on matching two-dimensional schemes for fractures167 with three-dimensional schemes for blocks. The numer-168 ical modeling of the second kind is based on pore-scale169 models of two-phase flow: ${ }^{16},{ }^{24},{ }^{27}$. In all these papers,, 170 the Navier-Stokes and Cahn-Hilliard equations were used 171 (the diffuse interface method). The results obtained in all172 these studies converge regarding the role of the capillary173 imbibition on the oil recovery: the higher the capillary 174 forces, the higher the imbibition effect and the ultimate175 oil recovery, ${ }^{17},{ }^{18},{ }^{24} . \mathrm{In}^{24}$ the capillary forces were varied by the surface tension, $\mathrm{in}^{16}$ by the flow rate, while in ${ }^{18}$ by injecting foams. However, the results remain contradic-176 tory regarding the dependence of the ultimate recovery on the injection rate. $\mathrm{In}^{16},{ }^{18},{ }^{24},{ }^{25}$ the ultimate recovery177 decreases with the injection rate, whilst in ${ }^{17},{ }^{20}$ the result is inverse. This indicates that the general physical ${ }_{178}$ theory of two-flow in double porosity media remains $\mathrm{an}_{179}$ open problem.

However, the development of such a theory is possible.181 More exactly, it is possible to develop the completely averaged model of such systems, in which nonlocality and nonlinearity are superimposed. The idea of such a method consists of spreading nonlinearity and nonlocality into different levels of the asymptotic expansion, that 182 is, to weaken their interaction. Such a splitting can be183 performed in so-called media with moderately contrast-184 ing properties.

On the basis of this idea, in the present work, we con-186 struct a completely averaged model of two-phase com-187 pressible flow, which combines elements of single-phase ${ }^{188}$ compressible and two-phase incompressible systems. We ${ }^{189}$ show that the interference between the capillarity and ${ }^{190}$ compressibility is more complicated than a simple sum ${ }^{191}$ of the saturation and pressure delay, but leads to more ${ }^{192}$ complicated cross effects:

- the effect of saturation delay caused by asymmetric ${ }^{194}$ extrusion of phases from the blocks due to the expansion and compaction of pores;

- the effect of saturation delay caused by nonlinear ${ }_{195}$ overlap of compressibility and capillarity.

All characteristic times have been obtained explicitly as the functions of saturation.
We applied the homogenization method based on twoscale asymptotic expansion in variational form, which is technically close to the constructive part of the two-scale convergence method ${ }^{3}$. It enables us to present all the calculations in the most compact form. This method is frequently applied to heterogeneous media. We extended the technique of the method to double porosity media, so that the method enables us to obtain the averaged equations both in fractures and blocks, and to remove macroscopic variables in the cell problem for the delay operator. This is why we prefer to explain the method in the main body of the paper, but not to place it in the appendix.

We underline that the paper does not study "deformable" media, but only "compressible" media, i.e. the media in which the porosity and permeability depend on pressure.

We also note that the model of periodic double porosity medium may be applied even for randomly heterogeneous media. This is shown, for instance, $i^{27}$, where a twophase flow was calculated on the pore scale. It was shown that the width of fingers (i.e. the width of fractures) and the distance between fingers (i.e. the width of blocks) are monotonically decreasing functions of the variance of the permeability field.

\section{PROBLEM FORMULATION}

\section{A. Equations of two-phase compressible flow}

Compressibility means that the density of liquids and the porosity/permeability of the medium depend on pressure. Traditionally, one uses the exponential laws for liquid and tight rocks:

$$
\frac{d \rho_{w}}{d p}=\widetilde{C}_{w} \rho_{w}, \quad \frac{d \rho_{o}}{d p}=\widetilde{C}_{o} \rho_{o}, \quad \frac{d \phi}{d p}=\widetilde{C}_{\phi} \phi
$$

where $p$ is the pressure, $\widetilde{C}_{w}, \widetilde{C}_{o}$, and $\widetilde{C}_{\phi}$ are the isothermal compressibility coefficients, which are considered to be constant and positive. Their dimension is $\mathrm{Pa}^{-1}$. The characteristic values of this parameter are discussed further in section VIIA. The positive derivative $d \phi / d p$ means that the porosity decreases if the pressure of liquid in the pores drops, which corresponds to the pore compaction under the weight of superimposed rocks.

For single-phase compressible fluids in compressible medium, one uses the classical simplification of flow equations, which consists in the following. Let us substitute the equation of compressibility (1) in the mass conservation law:

$$
\partial_{t}(\rho \phi)+\nabla \cdot(\rho \mathbf{V})=0
$$

where $\mathbf{V}$ is the Darcy velocity. Then we obtain:

$$
0=\frac{d(\rho \phi)}{d p} \partial_{t} p+\rho \nabla \cdot \mathbf{V}+\frac{d \rho}{d p} \mathbf{V} \cdot \nabla p
$$


The last term, $\mathbf{V} \cdot \nabla p \sim(\nabla p)^{2}$ is negligible, since the 231 gradients of pressure are very low in geological porous232 reservoirs. Then we obtain the classical equation of $_{233}$ single-phase compressible flow in compressible porous media:

$$
\phi C \partial_{t} p+\nabla \cdot \mathbf{V}=0, \quad C \equiv \frac{1}{\rho \phi} \frac{d(\rho \phi)}{d p}=\widetilde{C}_{w}+\widetilde{C}_{\phi}
$$

Let us apply the same assumption for two-phase fluids. ${ }^{23}$ Consider the immiscible phases and call them "water" ${ }_{239}$ and "oil". Each phase has its own pressure: $p_{w}$ and $p_{o \cdot 240}$ Note that $\rho_{w}=\rho_{w}\left(p_{w}\right), \rho_{o}=\rho_{o}\left(p_{o}\right)$, and $\phi=\phi\left(p_{w}\right)$ for $_{241}$ the pores occupied by water, or $\phi=\phi\left(p_{o}\right)$ for the pores occupied by oil.

The initial equations of mass conservation of the phases ${ }_{242}$ are:

$$
\partial_{t}\left(\rho_{w} \phi s\right)+\nabla \cdot\left(\rho_{w} \mathbf{V}_{w}\right)=0
$$

and

$$
\partial_{t}\left(\rho_{o} \phi(1-s)\right)+\nabla \cdot\left(\rho_{o} \mathbf{V}_{o}\right)=0
$$

where $s$ is the volume fraction of water ("the saturation"); $\rho$ is the phase density; $\phi$ is the medium porosity; $p$ is the pressure; $\mathbf{V}$ is the Darcy velocity vector. Indices $w$ and $o$ mean water and oil. Differentiating by parts, we obtain for water:

$\rho_{w} \phi \partial_{t} s+\frac{d\left(\rho_{w} \phi\right)}{d p_{w}} s \partial_{t} p_{w}+\rho_{w} \nabla \cdot \mathbf{V}_{w}+\frac{d \rho_{w}}{d p_{w}} \mathbf{V} \cdot \nabla p_{w}=0$

Neglecting the terms of the order $\sim(\nabla p)^{2}$, we finally obtain the equations of two-pase compressible flow in a porous medium

$$
\left\{\begin{array}{l}
\phi \partial_{t} s+\phi C_{w} s \partial_{t} p_{w}+\nabla \cdot \mathbf{V}_{w}=0 \\
-\phi \partial_{t} s+\phi C_{o}(1-s) \partial_{t} p_{o}+\nabla \cdot \mathbf{V}_{o}=0
\end{array}\right.
$$

where $C_{w} \equiv \widetilde{C}_{w}+\widetilde{C}_{\phi}, C_{o} \equiv \widetilde{C}_{o}+\widetilde{C}_{\phi}$.

This system is complemented by the equations of conservation of momentum in the form of Darcys law for each phase and the equation of capillary equilibrium, which relates the phase pressures:

$$
\begin{array}{lr}
\mathbf{V}_{\alpha}=-K \lambda_{\alpha}(s) \nabla p_{\alpha}, \quad \lambda_{\alpha}(s) \equiv \frac{k_{\alpha}(s)}{\mu_{\alpha}}, & \alpha=w, o \\
p_{o}=p_{w}+p_{c}(s) & (9 \mathrm{a})^{254} \\
{ }^{255} & (9 \mathrm{~b})^{256}
\end{array}
$$

where $K$ is the absolute permeability of the medium; $\mu^{258}$ is the dynamic viscosity of the phase. The functions of 259 relative phase permeability $k_{w}(s)$ and $k_{o}(s)$, and the cap-260 illary pressure $p_{c}(s)$ are given. They have the following ${ }^{261}$ properties:

- $k_{w}(s)$ is continuous monotonically increasing func-26 tion of water saturation $s$, such that $k_{w} \equiv 0$ for 265 $s \in\left[0, s_{*}\right]$, and $k_{w}(1)=1$;
- $k_{o}(s)$ is continuous monotonically decreasing function of $s$, such that $k_{o}(0)=1$ and $k_{o} \equiv 0$ for $s \in\left[s^{*}, 1\right]$;

- $p_{c}(s)$ is monotonically decreasing, such that $p_{c}(1)=0$ and $p_{c} \rightarrow \infty$ for $s \rightarrow s_{*}$. It is undefined for $s \in\left[0, s_{*}\right]$;

The values $s_{*}$ and $1-s_{*}$ are called the percolation thresholds or residual saturations. They mark the critical water and oil saturation below which the corresponding phase becomes immobile in porous medium. An example of these functions is given in Fig. 2.

\section{AVERAGING PROBLEM}

Let the flow domain be a bounded, connected region with a periodic microstructure of a characteristic scale $\varepsilon$, which is the ratio of the length of a period of heterogeneity to the length of the entire region. Parameter $\varepsilon$ is small: $0<\varepsilon \ll 1$ (Fig. 1a).
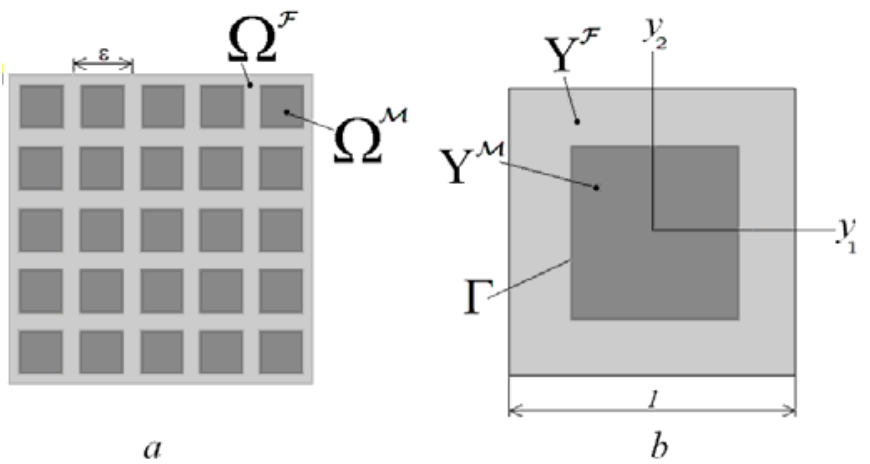

FIG. 1. Medium structure (a) and a single cell (b)

Four equations of mass and momentum conservation (8) and (9) can be reduced to two equations, excluding the Darcy velocities:

$$
\phi^{\varepsilon} \partial_{t} s_{\alpha}^{\varepsilon}+\phi^{\varepsilon} C_{\alpha}^{\varepsilon} s_{\alpha}^{\varepsilon} \partial_{t} p_{\alpha}^{\varepsilon}=\nabla \cdot\left(K^{\varepsilon} \lambda_{\alpha}^{\varepsilon} \nabla p_{\alpha}^{\varepsilon}\right)
$$

where $s_{o}^{\varepsilon} \equiv 1-s_{w}^{\varepsilon}, \quad \lambda_{\alpha}^{\varepsilon}(x, s)$ is defined in (9a).

The system (10) and (9b) defines three unknown functions: water saturation $s_{w}^{\varepsilon}$, pressure in water $p_{w}^{\varepsilon}$, and pressure in oil $p_{o}^{\varepsilon}$.

At the interface between the media, the normal phase fluxes and the phase pressures are continuous.

The boundary conditions specify the water pressure and saturation. The initial conditions fixes a specified saturation and pressure distribution in the domain: $s^{\varepsilon}(x, 0)=s^{0}(x)$ and $p_{w}^{\varepsilon}(x, 0)=p_{w}^{0}(x)$. If these initial values do not satisfy the condition of local capillary equilibrium, then the stated problem describes the relaxation of the initially non-equilibrium system to an equilibrium state. 


\section{A. Parameters of the problem}

Porosity, compressibility factors, phase permeability, and capillary pressure are different in blocks and frac- ${ }^{305}$ tures, but remain of the same order:

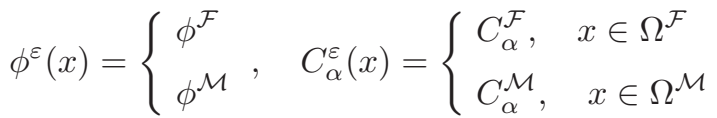

$\lambda_{\alpha}^{\varepsilon}(s, x)=\left\{\begin{array}{l}\lambda_{\alpha}^{\mathcal{F}}(s) \\ \lambda_{\alpha}^{\mathcal{M}}(s)\end{array}, \quad p_{c}^{\varepsilon}(s, x)= \begin{cases}p_{c}^{\mathcal{F}}(s), & x \in \Omega^{\mathcal{F}} \\ p_{c}^{\mathcal{M}}(s), & x \in \Omega^{\mathcal{M}}\end{cases}\right.$

where $\alpha=w, o$.

Parameters $\phi^{\mathcal{F}}, \phi^{\mathcal{M}}, C_{\alpha}^{\mathcal{F}}, C_{\alpha}^{\mathcal{M}}, \lambda_{\alpha}^{\mathcal{F}}(s), \lambda_{\alpha}^{\mathcal{M}}(s), p_{c}^{\mathcal{F}}(s){ }_{318}^{317}$ $p_{c}^{\mathcal{M}}(s)$ do not depend on $\varepsilon$. Parameters $\left.\phi^{\mathcal{F}}, \phi^{\mathcal{M}}, C_{\alpha}^{\mathcal{F}}\right)_{319}$ $C_{\alpha}^{\mathcal{M}}$ are positive. Functions $\left.\lambda_{\alpha}^{\mathcal{F}}(s), \lambda_{\alpha}^{\mathcal{M}}(s), p_{c}^{\mathcal{F}}(s)\right)_{320}$ $p_{c}^{\mathcal{M}}(s)$ are non-negative. We assume that $p_{c}^{\mathcal{M}}(s) \geq_{321}$ $p_{c}^{\mathcal{F}}(s)$, for any $s$, because the capillary pressure is higher ${ }_{322}$ when the permeability is lower.

At the same time, the absolute permeability of blocks 324 and fractures can be very different. It is known that 325 the permeability changes by several orders of magnitude 326 when the porosity varies within $0.1-0.2$. Therefore, we 327 accept the following basic condition of double porosity: 328

$$
K^{\varepsilon}(x)= \begin{cases}K^{\mathcal{F}}, & x \in \Omega^{\mathcal{F}} \\ \delta K^{\mathcal{M}}, & x \in \Omega^{\mathcal{M}}\end{cases}
$$

Coefficients $K^{\mathcal{F}}$ and $K^{\mathcal{M}}$ are positive and independent ${ }_{332}^{331}$ of $\varepsilon$.

Parameter $\delta$ is the degree of the contrast between the permeabilities of blocks and fractures. If the blocks are ${ }^{333}$ much less permeable than fractures, then this causes a delay/memory. The delay rate (or the memory length) ${ }_{334}$ can ne measured by another parameter, $\omega$, defined as:

$$
\omega=\frac{\varepsilon^{2}}{\delta}
$$

Two types of media are of interest, regarding the ${ }^{340}$ trast degree and the memory length ${ }^{13}$ :

- media with long memory (or strong contrast): $\omega \sim 1$ or $\delta \sim \varepsilon^{2}$. They are also called $\varepsilon^{2}$-media;

- media with short memory (or moderate contrast):342 $\varepsilon \ll \omega \ll 1$ or $\varepsilon^{2} \ll \delta \ll \varepsilon$.

There are also non-contrast media: $\delta \sim 1$, and media with impermeable blocks: $\delta \ll \varepsilon^{2}$. In the first case, the exchange process between blocks and fractures is without delay $\left(\omega \sim \varepsilon^{2} \ll 1\right)$, while it is completely absent in the second one due to a very strong delay $(\omega \gg 1)$. Both 344 these cases are of no interest for our research purposes. ${ }_{345}$

\section{METHOD OF HOMOGENIZATION WITH SPLITTING NONLOCALITY AND NONLINEARITY}

\section{A. The idea of the method}

The purpose of this paper is to obtain a completely averaged model. This concept was introduced in ${ }^{7}$. A model is called completely averaged, if its macroscopic equations do not contain microscopic variables, and the cell problem, which determines macroscopic coefficients, does not depend on macroscopic variables and, therefore, is solved only once.

To avoid the superimposition of the nonlinearity and the nonlocality, the idea is to spread them into different levels of asymptotic expansion over the parameter of memory length $\omega$, that is, nonlinearity is preserved in terms of zero order, whilst the nonlocality is allowed only in the first order. Since in asymptotic expansions the problem for the first approximation is usually linear, this enables us to treat the nonlocality completely and explicitly. For incompressible fluids, this was done in ${ }^{13}$ for the so-called moderate contrast between blocks and fractures.

This idea can be realized by considering $\omega$ as a small parameter and developing the solution of our problem into the asymptotic series over $\omega$. Therefore, we necessarily deal with media of moderate contrast in the permeability of block and fractures. For the sake of simplicity, we assume that

$$
\omega \sim \sqrt{\varepsilon}, \quad \text { or } \quad \delta=\varepsilon \sqrt{\varepsilon}
$$

which was used in ${ }^{13}$. Then, the total asymptotic expansion over $\varepsilon$ and $\omega$ reduces to a single expansion over $\varepsilon$ but which should include the fractional powers of $\varepsilon$.

\section{B. Two-scale formulation}

First of all, let us introduce the new variable $y$ that belongs to the unit cell of the microstructure, $Y=(0,1)^{d}$, which is shown in Fig. 1b, where $d$ is the space dimension. Domain $Y$ consists of two subdomains: a connected subdomain $Y^{\mathcal{F}}$ (Fractures) and $Y^{\mathcal{M}}$ (Matrix block). We denote by $\Gamma$ the boundary between the two subdomains in $Y$. Let us introduce the solution extension $\widetilde{s}^{\varepsilon}(x, y, t)$, $\widetilde{p}_{\alpha}^{\varepsilon}(x, y, t)$, where $y \in Y$, so that

$$
s^{\varepsilon}(x, t)=\left.\widetilde{s}^{\varepsilon}(x, y, t)\right|_{y=\frac{x}{\varepsilon}}, \quad p_{\alpha}^{\varepsilon}(x, t)=\left.\widetilde{p}_{\alpha}^{\varepsilon}(x, y, t)\right|_{y=\frac{x}{\varepsilon}}
$$

Then the following is true for the derivatives:

$$
\frac{\partial f(x, t)}{\partial x_{i}} \rightarrow\left(\frac{\partial \widetilde{f}(x, y, t)}{\partial x_{i}}+\frac{1}{\varepsilon} \frac{\partial \widetilde{f}(x, y, t)}{\partial y_{i}}\right)
$$

where $f$ is any function from $s^{\varepsilon}, p_{w}^{\varepsilon}, p_{o}^{\varepsilon}$

Since the operations of extension and differentiation commute, we find that the argument $y$ can be considered 
as an independent variable of $x$, and we should put it t374 $_{4}$ equal to $x / \varepsilon$ in final results. This is the main idea of $_{375}$ the two-scale homogenization method. The variable $x$ is called "slow", while $y$ is "fast".

Further, we will omit tilde.

The two-scale formulation of the problem (10) has the following form:

$$
\left\{\begin{array}{c}
\phi^{\varepsilon}(y) \partial_{t} s_{\alpha}^{\varepsilon}+\phi^{\varepsilon}(y) C_{\alpha}^{\varepsilon}(y) s_{\alpha}^{\varepsilon} \partial_{t} p_{\alpha}^{\varepsilon}= \\
\left(\partial_{x i}+\frac{1}{\varepsilon} \partial_{y i}\right)\left(K^{\varepsilon}(y) \lambda_{\alpha}^{\varepsilon}\left(\partial_{x i} p_{\alpha}^{\varepsilon}+\frac{1}{\varepsilon} \partial_{y i} p_{\alpha}^{\varepsilon}\right)\right), \\
x \in \Omega, \quad y \in Y, \quad t \in(0, T) \\
p_{o}^{\varepsilon}=p_{w}^{\varepsilon}+p_{c}^{\varepsilon}, \quad s_{o}^{\varepsilon}=1-s_{w}^{\varepsilon}
\end{array}\right.
$$

with conditions:

$$
\left\{\begin{array}{l}
\left.s_{w}^{\varepsilon}\right|_{t=0}=s^{0}(x),\left.\quad p_{w}^{\varepsilon}\right|_{t=0}=p_{w}^{0}(x) \\
{\left[K^{\varepsilon} \lambda_{\alpha}^{\varepsilon} \frac{\partial p_{\alpha}^{\varepsilon}}{\partial n}\right]_{\Gamma}=0, \quad\left[p_{\alpha}^{\varepsilon}\right]_{\Gamma}=0, \quad \alpha=w, o} \\
s_{w}^{\varepsilon}, p_{w}^{\varepsilon} \text { are } y \text {-periodic }
\end{array}\right.
$$

where [.] means a jump. Note that the continuity of phase pressures means the continuity of the capillary pressure: $\left[p_{c}^{\varepsilon}\right]=0$, which determine a discontinuity of the saturation $s^{\varepsilon}$.

It can be presented in variational form:

$$
\begin{aligned}
& \int_{\Omega} \int_{Y} \zeta \phi^{\varepsilon} \partial_{t} s_{\alpha}^{\varepsilon} d x d y+\int_{\Omega} \int_{Y} \zeta \phi^{\varepsilon} C_{\alpha}^{\varepsilon} s_{\alpha}^{\varepsilon} \partial_{t} p_{\alpha}^{\varepsilon} d x d y= \\
& -\frac{1}{\varepsilon^{2}} \int_{\Omega} \int_{Y^{\mathcal{F}}} K^{\mathcal{F}} \lambda_{\alpha}^{\mathcal{F}}\left(\partial_{y i} p_{\alpha}^{\varepsilon}+\varepsilon \partial_{x i} p_{\alpha}^{\varepsilon}\right)\left(\partial_{y i} \zeta+\varepsilon \partial_{x i} \zeta\right) d y d x- \\
& \frac{1}{\sqrt{\varepsilon}} \int_{\Omega} \int_{Y^{\mathcal{M}}} K^{\mathcal{M}} \lambda_{\alpha}^{\mathcal{M}}\left(\partial_{y i} p_{\alpha}^{\varepsilon}+\varepsilon \partial_{x i} p_{\alpha}^{\varepsilon}\right)\left(\partial_{y i} \zeta+\varepsilon \partial_{x i} \zeta\right) d y d x
\end{aligned}
$$

for any function $\zeta(x, y)$ in $\Omega \times Y$, such that $\zeta$ is continuous, $\left.\zeta\right|_{\partial \Omega}=0$ and is periodic with respect to $y$.

The relationship (20) is obtained by multiplying (18) by $\zeta$, integrating by parts, and using the GaussOstrogradsky theorem. The integrals over the boundary $\partial \Omega$ are zero due to the fact that the function $\zeta$ is zero on $\partial \Omega$. The integrals over the boundary $\partial Y$ of the period $Y$ are zero due to the periodicity of all functions with respect to $y$.

\section{Asymptotic expansion}

Homogenization is performed by the method of twoscale asymptotic expansions with respect to parameter ${ }^{388}$ $\varepsilon$, as well as regular asymptotic series with respect to the nonlocality parameter $\omega$, which means, given (15), the 389 appearance of terms containing fractional powers $\varepsilon^{1 / 2} .390$
The general structure of the expansion is as follows, for $\alpha=w, o$ :

$$
\begin{aligned}
& p_{\alpha}^{\varepsilon}(x, y, t)= \\
& \left\{\begin{array}{l}
p_{\alpha 0}(x, t)+\sqrt{\varepsilon} p_{\alpha^{1} / 2}^{\mathcal{M}}(x, y, t)+\varepsilon p_{\alpha 1}^{\mathcal{M}}(x, y, t)+\ldots, \quad y \in Y^{\mathcal{M}} \\
p_{\alpha 0}(x, t)+\sqrt{\varepsilon} p_{\alpha^{1} / 2}^{\mathcal{F}}(x, t)+\varepsilon p_{\alpha 1}^{\mathcal{F}}(x, y, t)+\ldots, \quad y \in Y^{\mathcal{F}}
\end{array}\right.
\end{aligned}
$$

$$
\begin{aligned}
& s_{\alpha}^{\varepsilon}(x, y, t)= \\
& \left\{\begin{array}{l}
s_{\alpha 0}(x, t)+\sqrt{\varepsilon} s_{\alpha^{1 / 2}}^{\mathcal{M}}(x, y, t)+\varepsilon s_{\alpha 1}^{\mathcal{M}}(x, y, t)+\ldots, \quad y \in Y^{\mathcal{M}} \\
s_{\alpha 0}(x, t)+\sqrt{\varepsilon} s_{\alpha^{1 / 2}}^{\mathcal{F}}(x, t)+\varepsilon s_{\alpha 1}^{\mathcal{F}}(x, y, t)+\ldots, \quad y \in Y^{\mathcal{F}}
\end{array}\right.
\end{aligned}
$$

In addition, the condition of continuity of phase pressures holds:

$$
\left.p_{\alpha^{1} / 2}^{\mathcal{M}}(x, y, t)\right|_{y \in \Gamma}=p_{\alpha^{1} / 2}^{\mathcal{F}}(x, t)
$$

The independence of the zero terms of $y$ is easy to prove by substituting the asymptotic expansion into (20). For nonlinear functions in (20), the expansion is as follows:

$$
\lambda_{\alpha}^{\varepsilon}= \begin{cases}\lambda_{\alpha 0}(x, t)+\sqrt{\varepsilon} \lambda_{\alpha^{1} / 2}^{\mathcal{M}}(x, y, t)+\varepsilon \ldots, & y \in Y^{\mathcal{M}} \\ \lambda_{\alpha 0}(x, t)+\sqrt{\varepsilon} \lambda_{\alpha^{1 / 2}}^{\mathcal{F}}(x, t)+\varepsilon \ldots, & y \in Y^{\mathcal{F}}\end{cases}
$$

Then the integral identity (20) takes the form:

$$
\begin{aligned}
& \int_{\Omega} \int_{Y} \zeta \phi^{\varepsilon}\left(\partial_{t} s_{\alpha 0}+\sqrt{\varepsilon} \partial_{t} s_{\alpha^{1 / 2}}\right) d x d y+ \\
& \int_{\Omega} \int_{Y} \zeta \phi^{\varepsilon} C_{\alpha}^{\varepsilon}\left(s_{\alpha 0}+\sqrt{\varepsilon} s_{\alpha^{1 / 2} / 2}\right)\left(\partial_{t} p_{\alpha 0}+\sqrt{\varepsilon} \partial_{t} p_{\alpha 1 / 2}\right) d x d y= \\
& -\frac{1}{\varepsilon} \int_{\Omega} \int_{Y^{\mathcal{F}}} K^{\mathcal{F}}\left(\lambda_{\alpha 0}^{\mathcal{F}}+\sqrt{\varepsilon} \lambda_{\alpha^{1} / 2}^{\mathcal{F}}\right)\left(\partial_{y i} p_{\alpha 1}+\partial_{x i} p_{\alpha 0}+\right. \\
& \left.\sqrt{\varepsilon}\left(\partial_{y i} p_{\alpha^{3} / 2}+\partial_{x i} p_{\alpha^{1} / 2}\right)\right)\left(\partial_{y i} \zeta+\varepsilon \partial_{x i} \zeta\right) d y d x- \\
& -\int_{\Omega} \int_{Y^{\mathcal{M}}} K^{\mathcal{M}} \lambda_{\alpha 0}^{\mathcal{M}}\left(\partial_{y i} p_{\alpha^{1} / 2}+\sqrt{\varepsilon}\left(\partial_{y i} p_{\alpha 1}+\partial_{x i} p_{\alpha 0}\right)\right) \times \\
& \left(\partial_{y i} \zeta+\varepsilon \partial_{x i} \zeta\right) d y d x+\mathcal{O}(\varepsilon)
\end{aligned}
$$

The homogenization technique consists of substituting the asymptotic expansion into the integral identity (24) and obtaining closed expressions for subsequent terms of the expansion by selecting various types of test functions $\zeta$.

\section{Result of homogenization: macroscopic model}

We immediately give the result of homogenization. Its derivation is given in the next section. 
Let us define the averaged phase saturations and pres-405 sures in the blocks and fractures as follows:

$$
\begin{aligned}
& \mathcal{P}_{\alpha}^{\mathcal{F}} \equiv \frac{1}{\left|Y^{\mathcal{F}}\right|} \int_{Y^{\mathcal{F}}}\left(p_{\alpha 0}+\sqrt{\varepsilon} p_{\alpha^{1 / 2}}^{\mathcal{F}}\right) d y=p_{\alpha 0}+\sqrt{\varepsilon} p_{\alpha^{1 / 2}}^{\mathcal{F}}, \\
& \mathcal{P}_{\alpha}^{\mathcal{M}} \equiv \frac{1}{\left|Y^{\mathcal{M}}\right|} \int_{Y^{\mathcal{M}}}\left(p_{\alpha 0}+\sqrt{\varepsilon} p_{\alpha^{1} / 2}^{\mathcal{M}}\right) d y= \\
& p_{\alpha 0}+\sqrt{\varepsilon}\left\langle p_{\alpha^{1 / 2}}^{\mathcal{M}}\right\rangle_{\mathcal{M}}, \\
& \mathcal{S}_{\alpha}^{\mathcal{F}} \equiv \frac{1}{\left|Y^{\mathcal{F}}\right|} \int_{Y^{\mathcal{F}}}\left(s_{\alpha 0}+\sqrt{\varepsilon} s_{\alpha^{1} / 2}^{\mathcal{F}}\right) d y=s_{\alpha 0}+\sqrt{\varepsilon} s_{\alpha^{1} / 2}^{\mathcal{F}}, \\
& \mathcal{S}_{\alpha}^{\mathcal{M}} \equiv \frac{1}{\left|Y^{\mathcal{M}}\right|} \int_{Y^{\mathcal{M}}}\left(s_{\alpha 0}+\sqrt{\varepsilon} s_{\alpha^{1 / 2}}^{\mathcal{M}}\right) d y= \\
& s_{\alpha 0}+\sqrt{\varepsilon}\left\langle s_{\alpha^{1 / 2}}^{\mathcal{M}}\right\rangle_{\mathcal{M}}
\end{aligned}
$$

where $\langle\cdot\rangle_{i}=\frac{1}{\left|Y^{i}\right|} \int_{Y^{i}}(\cdot) d y, \quad i=\mathcal{F}, \mathcal{M}$

The following links exist between them:

$$
\begin{aligned}
& \mathcal{S}_{o}^{\mathcal{F}}=1-\mathcal{S}_{w}^{\mathcal{F}}, \quad \mathcal{S}_{o}^{\mathcal{M}}=1-\mathcal{S}_{w}^{\mathcal{F}}, \\
& \mathcal{P}_{o}^{\mathcal{F}}=\mathcal{P}_{w}^{\mathcal{F}}+p_{c}^{\mathcal{F}}\left(\mathcal{S}_{w}^{\mathcal{F}}\right), \quad \mathcal{P}_{o}^{\mathcal{M}}=\mathcal{P}_{w}^{\mathcal{M}}+p_{c}^{\mathcal{M}}\left(\mathcal{S}_{w}^{\mathcal{M}}\right)
\end{aligned}
$$

Note that macroscopic capillary pressures in fractures and blocks, $\mathcal{P}_{c}^{\mathcal{F}}\left(\mathcal{S}_{w}^{\mathcal{F}}\right)$ and $\mathcal{P}_{c}^{\mathcal{M}}\left(\mathcal{S}^{\mathcal{M}}\right)$, do not figure in this model, since they are simply equal to the original capillary pressure functions taken from the average saturations, with accuracy $\mathcal{O}(\varepsilon)$ :

$$
\mathcal{P}_{c}^{\mathcal{F}}\left(\mathcal{S}_{w}^{\mathcal{F}}\right)=p_{c}^{\mathcal{F}}\left(\mathcal{S}_{w}^{\mathcal{F}}\right), \quad \mathcal{P}_{c}^{\mathcal{M}}\left(\mathcal{S}_{w}^{\mathcal{M}}\right)=p_{c}^{\mathcal{M}}\left(\mathcal{S}_{w}^{\mathcal{M}}\right)
$$

The macroscopic model has the form of the following system of equations for the average pressures and satu-414 rations in the blocks and fractures:

$$
\begin{gathered}
\phi^{\mathcal{F}}(1-\theta) \partial_{t} \mathcal{S}_{\alpha}^{\mathcal{F}}+\phi^{\mathcal{F}} C_{\alpha}^{\mathcal{F}}(1-\theta) \mathcal{S}_{\alpha}^{\mathcal{F}} \partial_{t} \mathcal{P}_{\alpha}^{\mathcal{F}}= \\
\partial_{x i}\left(\mathbb{K}_{i k} \lambda_{\alpha}^{\mathcal{F}} \partial_{x i} \mathcal{P}_{\alpha}^{\mathcal{F}}\right)+\xi_{\alpha}\left(\mathcal{P}_{\alpha}^{\mathcal{M}}-\mathcal{P}_{\alpha}^{\mathcal{F}}\right), \quad \alpha=w, o \\
\mathcal{P}_{w}^{\mathcal{M}}=\mathcal{P}_{w}^{\mathcal{F}}-\frac{\tau_{w}^{\mathrm{com}}}{C_{w}^{\mathcal{M}} \mathcal{S}_{w}^{\mathcal{M}}}\left[\partial_{t} \mathcal{S}_{w}^{\mathcal{M}}+C_{w}^{\mathcal{M}} \mathcal{S}_{w}^{\mathcal{M}} \partial_{t} \mathcal{P}_{w}^{\mathcal{M}}\right] \\
p_{c}^{\mathcal{M}}\left(\mathcal{S}_{w}^{\mathcal{M}}\right)=p_{c}^{\mathcal{F}}+\frac{\tau^{\mathrm{cap}}+\tau^{\mathrm{cc}}}{C_{o}^{\mathcal{M}}} \partial_{t} \mathcal{S}_{w}^{\mathcal{M}}+ \\
\left(\tau_{w}^{\mathrm{com}}-\tau_{o}^{\mathrm{com}}\right) \partial_{t} \mathcal{P}_{w}^{\mathcal{M}}
\end{gathered}
$$

where $\theta$ is the volume fractions of the blocks.

The effective permeability is defined as:

$$
\mathbb{K}_{i k} \equiv \int_{Y^{\mathcal{F}}} K^{\mathcal{F}}\left(\partial_{y i} \psi_{k}+\delta_{i k}\right) d y
$$

and the cell functions $\psi_{k}(y)$ are the solution to the first problem in a cell (in the fracture), for $k=1,2,3$ :

$$
\left\{\begin{array}{l}
\partial_{y i}\left(K^{\mathcal{F}}\left(\partial_{y i} \psi_{k}+\delta_{i k}\right)\right)=0, \quad y \in Y^{\mathcal{F}} \\
\left.K^{\mathcal{F}}\left(\partial_{y i} \psi_{k}+\delta_{i k}\right) n_{i}^{\Gamma}\right|_{y \in \Gamma}=0, \\
\psi_{k} \text { is } y \text { - periodic } \\
\left\langle\psi_{k}\right\rangle_{Y^{\mathcal{F}}}=0
\end{array}\right.
$$

The delay times between blocks and fractures caused by the phase compressibility are defined as:

$$
\tau_{\alpha}^{\text {com }}\left(\mathcal{S}_{w}^{\mathcal{M}}\right)=\sqrt{\varepsilon}\langle\varphi\rangle_{\mathcal{M}} \phi^{\mathcal{M}} \frac{C_{\alpha}^{\mathcal{M}} \mathcal{S}_{\alpha}^{\mathcal{M}}}{\lambda_{\alpha}^{\mathcal{M}}}, \quad \alpha=w, o
$$

The delay times caused by capillarity and joint action of capillarity and compressibility are as follows:

$$
\begin{aligned}
& \tau^{\text {cap }}\left(\mathcal{S}_{w}^{\mathcal{M}}\right)=\sqrt{\varepsilon}\langle\varphi\rangle_{\mathcal{M}} \phi^{\mathcal{M}} C_{o}^{\mathcal{M}} \frac{\left(\lambda_{w}^{\mathcal{M}}+\lambda_{o}^{\mathcal{M}}\right)}{\lambda_{w}^{\mathcal{M}} \lambda_{o}^{\mathcal{M}}}, \\
& \tau^{\mathrm{cc}}\left(\mathcal{S}_{w}^{\mathcal{M}}\right)=-C_{o}^{\mathcal{M}} \frac{d p_{c}^{\mathcal{M}}}{d \mathcal{S}^{\mathcal{M}}} \tau_{o}^{\mathrm{com}}
\end{aligned}
$$

where the function $\varphi(y)$ is the solution of the second cell problem (in the block):

$$
\left\{\begin{array}{l}
\frac{\partial}{\partial y_{i}}\left(K^{\mathcal{M}} \frac{\partial \varphi}{\partial y_{i}}\right)=-1, \quad y \in Y^{\mathcal{M}} \\
\left.\varphi\right|_{y \in \Gamma}=0
\end{array}\right.
$$

The transfer functions $\xi_{\alpha}$ are defined as:

$$
\xi_{\alpha} \equiv \frac{\theta \lambda_{\alpha}^{\mathcal{M}}}{\sqrt{\varepsilon}\langle\varphi\rangle_{\mathcal{M}}}
$$

where $\theta$ is the volume fraction of blocks.

The model (28) is the formal asymptotic expansion of the original problem that keeps the terms of order $\mathcal{O}(\sqrt{\varepsilon})$. The order of residual terms is, thus, $\mathcal{O}(\varepsilon)$.

\section{DERIVATION OF THE AVERAGED MODEL}

\section{A. First step of homogenization: expansion in fractures}

Let $\zeta=\zeta(x, y)$ in $\Omega \times Y,\left.\zeta\right|_{\partial \Omega}=0, \zeta$ is continuous and periodic with respect to $y$. Then one obtains from (24) for negatives powers of $\varepsilon$ :

$$
\begin{aligned}
& 0=\frac{1}{\varepsilon} \int_{\Omega} \int_{Y^{\mathcal{F}}} K^{\mathcal{F}} \lambda_{\alpha 0}^{\mathcal{F}}\left(\partial_{y i} p_{\alpha 1}+\partial_{x i} p_{\alpha 0}\right) \partial_{y i} \zeta d y d x, \\
& 0=\frac{1}{\sqrt{\varepsilon}} \int_{\Omega} \int_{Y^{\mathcal{F}}} K^{\mathcal{F}}\left[\lambda_{\alpha 0}^{\mathcal{F}}\left(\partial_{y i} p_{\alpha 3 / 2}+\partial_{x i} p_{\alpha 1 / 2}\right)+\right. \\
& \left.\lambda_{\alpha 1 / 2}^{\mathcal{F}}\left(\partial_{y i} p_{\alpha 1}+\partial_{x i} p_{\alpha 0}\right)\right] \partial_{y i} \zeta d y d x+\mathcal{O}(\varepsilon)
\end{aligned}
$$




$$
\begin{gathered}
0=-\int_{\Omega} \int_{Y^{\mathcal{F}}} \zeta \partial_{y i}\left(K^{\mathcal{F}} \lambda_{\alpha 0}^{\mathcal{F}}\left(\partial_{y i} p_{\alpha 1}+\partial_{x i} p_{\alpha 0}\right)\right) d x d y+{ }^{437} \\
\int_{\Omega} \int_{\Gamma} \zeta K^{\mathcal{F}} \lambda_{\alpha 0}^{\mathcal{F}}\left(\partial_{y i} p_{\alpha 1}+\partial_{x i} p_{\alpha 0}\right) n_{i}^{\Gamma} d x d y
\end{gathered}
$$

$$
\begin{gathered}
0=\int_{\Omega} \int_{Y^{\mathcal{F}}} \zeta \partial_{y i}\left(K^{\mathcal{F}} \lambda_{\alpha 0}^{\mathcal{F}}\left(\partial_{y i} p_{\alpha^{3} / 2}+\partial_{x i} p_{\alpha^{1 / 2} / 2}\right)+\right. \\
\left.K^{\mathcal{F}} \lambda_{\alpha 1}^{\mathcal{F}}\left(\partial_{y i} p_{\alpha 1}+\partial_{x i} p_{\alpha 0}\right)\right) d y d x+ \\
\int_{\Omega} \int_{\Gamma} \zeta\left[K^{\mathcal{F}} \lambda_{\alpha 0}^{\mathcal{F}}\left(\partial_{y i} p_{\alpha^{3} / 2}+\partial_{x i} p_{\alpha^{1} / 2}\right)+\right. \\
\left.K^{\mathcal{F}} \lambda_{\alpha 1}^{\mathcal{F}}\left(\partial_{y i} p_{\alpha 1}+\partial_{x i} p_{\alpha 0}\right)\right] n_{i}^{\Gamma} d x d y
\end{gathered}
$$

Taking into account (23), this is equivalent to two problems in classical formulation:

$$
\left\{\begin{array}{l}
\partial_{y i}\left(K^{\mathcal{F}}\left(\partial_{y i} p_{\alpha 1}^{\mathcal{F}}+\partial_{x i} p_{\alpha 0}\right)\right)=0, \quad y \in Y^{\mathcal{F}} \\
\left.K^{\mathcal{F}}\left(\partial_{y i} p_{\alpha 1}^{\mathcal{F}}+\partial_{x i} p_{\alpha 0}\right) n_{i}^{\Gamma}\right|_{y \in \Gamma}=0
\end{array}\right.
$$

427

$$
\left\{\begin{array}{l}
\partial_{y i}\left(K^{\mathcal{F}}\left(\partial_{y i} p_{\alpha^{3} / 2}^{\mathcal{F}}+\partial_{x i} p_{\alpha^{1} / 2}\right)\right)=0, \quad y \in Y^{\mathcal{F}} \\
\left.K^{\mathcal{F}}\left(\partial_{y i} p_{\alpha^{3} / 2}^{\mathcal{F}}+\partial_{x i} p_{\alpha^{1} / 2}\right) n_{i}^{\Gamma}\right|_{y \in \Gamma}=0
\end{array}\right.
$$

This leads to the following representation of $p_{\alpha 1}^{\mathcal{F}}$ and $p_{\alpha^{3} / 2}^{\mathcal{F}}$ through $p_{\alpha 0}$ and $p_{\alpha^{1 / 2}}^{\mathcal{F}}$ :

$$
\begin{aligned}
& p_{\alpha 1}^{\mathcal{F}}=\psi_{k}(y) \frac{\partial p_{\alpha 0}}{\partial x_{k}}+\bar{p}_{\alpha 1}^{\mathcal{F}}(x, t), \\
& p_{\alpha^{3} / 2}^{\mathcal{F}}=\psi_{k}(y) \frac{\partial p_{\alpha^{1} / 2}^{\mathcal{F}}}{\partial x_{k}}+\bar{p}_{\alpha^{3} / 2}^{\mathcal{F}}(x, t)
\end{aligned}
$$

where $\bar{p}_{\alpha 1}^{\mathcal{F}}(x, t)$ and $\bar{p}_{\alpha^{3} / 2}^{\mathcal{F}}(x, t)$ are some slow functions, which do not enter in the homogenized model. For functions $\psi_{k}$, we obtain the first cell problem (30), in which the latter condition is added to ensure the solution uniqueness.

\section{B. Second step: the averaged equation in fractures}

Let us select the test functions as $\zeta=\zeta(x)$ in $\Omega$ and $\left.\zeta\right|_{\partial \Omega}=0$. Then one obtains from (24):

$$
\begin{aligned}
& \int_{\Omega} \int_{Y} \zeta \phi\left(\partial_{t} s_{\alpha 0}+\sqrt{\varepsilon} \partial_{t} s_{\alpha^{1 / 2} / 2}\right) d x d y+ \\
& \int_{\Omega} \int_{Y} \zeta \phi C_{\alpha}\left(s_{\alpha 0}+\sqrt{\varepsilon} s_{\alpha^{1 / 2} / 2}\right)\left(\partial_{t} p_{\alpha 0}+\sqrt{\varepsilon} \partial_{t} p_{\alpha^{1 / 2} / 2}\right) d x d y= \\
& -\int_{\Omega} \int_{Y^{\mathcal{F}}} K^{\mathcal{F}}\left(\lambda_{\alpha 0}^{\mathcal{F}}+\sqrt{\varepsilon} \lambda_{\alpha^{1} / 2}^{\mathcal{F}}\right)\left[\partial_{y i} p_{\alpha 1}+\partial_{x i} p_{\alpha 0}+\right. \\
& \left.\sqrt{\varepsilon}\left(\partial_{y i} p_{\alpha^{3} / 2}+\partial_{x i} p_{\alpha^{1} / 2}\right)\right] \partial_{x i} \zeta d y d x+\mathcal{O}(\varepsilon)
\end{aligned}
$$

Using (40) we deduce:

$$
\begin{aligned}
& \int_{\Omega} \int_{Y} \zeta \phi \partial_{t}\left(s_{\alpha 0}+\sqrt{\varepsilon} s_{\alpha^{1 / 2}}\right) d x d y+ \\
& \int_{\Omega} \int_{Y} \zeta \phi C_{\alpha}\left(s_{\alpha 0}+\sqrt{\varepsilon} s_{\alpha^{1 / 2} / 2}\right) \partial_{t}\left(p_{\alpha 0}+\sqrt{\varepsilon} p_{\alpha^{1 / 2} / 2}\right) d x d y= \\
& \int_{\Omega} \int_{Y^{\mathcal{F}}} \zeta \partial_{x i}\left(K^{\mathcal{F}}\left(\lambda_{\alpha 0}^{\mathcal{F}}+\sqrt{\varepsilon} \lambda_{\alpha^{1} / 2}^{\mathcal{F}}\right)\left(\partial_{y i} \psi_{k}+\delta_{i k}\right) \times\right. \\
& \left.\partial_{x k}\left(p_{\alpha 0}+\sqrt{\varepsilon} p_{\alpha 1 / 2}^{\mathcal{F}}\right)\right) d y d x+\mathcal{O}(\varepsilon)
\end{aligned}
$$

$$
\begin{aligned}
& \int_{\Omega} \zeta\left[\phi^{\mathcal{F}}(1-\theta) \partial_{t} \mathcal{S}_{\alpha}^{\mathcal{F}}+\phi^{\mathcal{M}} \theta \partial_{t} \mathcal{S}_{\alpha}^{\mathcal{M}}\right] d x+ \\
& \int_{\Omega} \zeta\left[\phi^{\mathcal{F}} C_{\alpha}^{\mathcal{F}}(1-\theta) \mathcal{S}_{\alpha}^{\mathcal{F}} \partial_{t} \mathcal{P}_{\alpha}^{\mathcal{F}}+\phi^{\mathcal{M}} C_{\alpha}^{\mathcal{M}} \theta \mathcal{S}_{\alpha}^{\mathcal{M}} \partial_{t} \mathcal{P}_{\alpha}^{\mathcal{M}}\right] d x= \\
& \int_{\Omega} \zeta \partial_{x i}\left(\mathbb{K}_{i k} \lambda_{\alpha}^{\mathcal{F}}\left(\mathcal{S}_{\alpha} \mathcal{F}\right) \partial_{x k} \mathcal{P}_{\alpha}^{\mathcal{F}}\right) d x
\end{aligned}
$$

where the effective permeability tensor is (29). This gives the first averaged equation (28a) in the as-yet-incomplete form:

$$
\begin{aligned}
& \phi^{\mathcal{F}}(1-\theta) \partial_{t} \mathcal{S}_{\alpha}^{\mathcal{F}}+\phi^{\mathcal{F}} C_{\alpha}^{\mathcal{F}}(1-\theta) \mathcal{S}_{\alpha}^{\mathcal{F}} \partial_{t} \mathcal{P}_{\alpha}^{\mathcal{F}}= \\
& \partial_{x i}\left(\mathbb{K}_{i k} \lambda_{\alpha}^{\mathcal{F}}\left(\mathcal{S}_{\alpha} \mathcal{F}\right) \partial_{x k} \mathcal{P}_{\alpha}^{\mathcal{F}}\right)-\phi^{\mathcal{M}} \theta \partial_{t} \mathcal{S}_{\alpha}^{\mathcal{M}}- \\
& \phi^{\mathcal{M}} C_{\alpha}^{\mathcal{M}} \theta \mathcal{S}_{\alpha}^{\mathcal{M}} \partial_{t} \mathcal{P}_{\alpha}^{\mathcal{M}}, \quad \alpha=w, o
\end{aligned}
$$




\section{Third step: expansion in blocks}

Let $\zeta=\zeta(x, y)$ in $\Omega \times Y,\left.\zeta\right|_{\partial \Omega}=0, \zeta \equiv 0$ in $Y^{\mathcal{F}}$, and $\zeta$ is continuous. Then the identity (24) gives:

$$
\begin{aligned}
& \int_{\Omega} \int_{Y^{\mathcal{M}}} \zeta \phi\left(\partial_{t} s_{\alpha 0}+\sqrt{\varepsilon} \partial_{t} s_{\alpha^{1 / 2}}^{\mathcal{M}}\right) d x d y+ \\
& \int_{\Omega} \int_{Y^{\mathcal{M}}} \zeta \phi C_{\alpha}\left(s_{\alpha 0}+\sqrt{\varepsilon} s_{\alpha^{1} / 2}^{\mathcal{M}}\right)\left(\partial_{t} p_{\alpha 0}+\sqrt{\varepsilon} \partial_{t} p_{\alpha^{1} / 2}^{\mathcal{M}}\right) d x d y= \\
& -\int_{\Omega_{Y^{\mathcal{M}}}} K^{\mathcal{M}}\left(\lambda_{\alpha 0}+\sqrt{\varepsilon} \lambda_{\alpha^{1 / 2}}^{\mathcal{M}}\right)\left[\partial_{y i} p_{\alpha^{1} / 2}+\right. \\
& \left.\sqrt{\varepsilon}\left(\partial_{y i} p_{\alpha 1}+\partial_{x i} p_{\alpha 0}\right)\right] \partial_{x i} \zeta d y d x+\mathcal{O}(\varepsilon)
\end{aligned}
$$

The integral over the block boundary is zero, since ${ }^{468}$ functions $w$ are zero in the fracture and are continuous. The zero-order terms yield:

$$
\begin{gathered}
\int_{\Omega} \int_{Y \mathcal{M}} \zeta \phi\left(\partial_{t} s_{\alpha 0}+C_{\alpha} s_{\alpha 0} \partial_{t} p_{\alpha 0}\right) d x d y= \\
\int_{\Omega} \int_{Y^{\mathcal{M}}} \zeta \partial_{y i}\left(K^{\mathcal{M}} \lambda_{\alpha 0} \partial_{y i} p_{\alpha 1 / 2}\right) d y d x
\end{gathered}
$$
(22):

$$
p_{\alpha^{1} / 2}^{\mathcal{M}}=p_{\alpha^{1} / 2}^{\mathcal{F}}-\varphi(y) \frac{\phi^{\mathcal{M}}}{\lambda_{\alpha 0}}\left(\partial_{t} s_{\alpha 0}+C_{\alpha}^{\mathcal{M}} s_{\alpha 0} \partial_{t} p_{\alpha 0}\right)
$$

where $\varphi(y)$ is he solution of the second cell problem (33). The boundary condition in (33) results from the continuity of the phase pressures (22).

\section{Fourth step: averaged equation in blocks}

Formula (47) enables us to obtain an explicit relation ${ }^{476}$ between $\mathcal{P}_{\alpha}^{\mathcal{M}}$ and $\mathcal{P}_{\alpha}^{\mathcal{F}}$. Indeed, taking the average of $(47)^{477}$ over $Y^{\mathcal{M}}$ (and noting that only the function $\phi$ depends $s^{478}$ on $y$ in the right-hand side of (47)), multiplying by $\sqrt{\varepsilon^{479}}$ and adding $p_{\alpha 0}$, we obtain:

$$
\begin{aligned}
p_{\alpha 0}+\sqrt{\varepsilon}\left\langle p_{\alpha^{1} / 2}^{\mathcal{M}}\right\rangle_{\mathcal{M}} & =p_{\alpha 0}+\sqrt{\varepsilon} p_{\alpha^{1 / 2}}^{\mathcal{F}}- \\
& \sqrt{\varepsilon}\langle\varphi\rangle_{\mathcal{M}} \frac{\phi^{\mathcal{M}}}{\lambda_{\alpha 0}}\left(\partial_{t} s_{\alpha 0}+C_{\alpha}^{\mathcal{M}} s_{\alpha 0} \partial_{t} p_{\alpha 0}\right)
\end{aligned}
$$

Using the definition of the averaged pressures and saturations (25), we deduce:

$\mathcal{P}_{\alpha}^{\mathcal{M}}=\mathcal{P}_{\alpha}^{\mathcal{F}}-\frac{\tau_{\alpha}^{\text {com }}}{C_{\alpha}^{\mathcal{M}} \mathcal{S}_{\alpha}^{\mathcal{M}}}\left(\partial_{t} \mathcal{S}_{\alpha}^{\mathcal{M}}+C_{\alpha}^{\mathcal{M}} \mathcal{S}_{\alpha}^{\mathcal{M}} \partial_{t} \mathcal{P}_{\alpha}^{\mathcal{M}}\right), \quad \alpha=w, o$

which is identical to $(28 \mathrm{~b})$. The characteristic times of delay are defined as (31).

In the structure of the delay times, we have taken into account the following circumstances:

$$
\begin{gathered}
\partial_{t} s_{\alpha 0}=\partial_{t}\left(s_{\alpha 0}+\sqrt{\varepsilon}\left\langle s_{\alpha, 01}^{\mathcal{M}}\right\rangle_{\mathcal{M}}\right)-\sqrt{\varepsilon} \partial_{t}\left\langle s_{\alpha, 01}^{\mathcal{M}}\right\rangle_{\mathcal{M}}= \\
\partial_{t} \mathcal{S}_{\alpha}^{\mathcal{M}}+\mathcal{O}(\sqrt{\varepsilon}) \\
\lambda_{\alpha 0}^{\mathcal{M}}=\lambda_{\alpha}^{\mathcal{M}}\left(s_{\alpha 0}\right)+\sqrt{\varepsilon} \frac{d \lambda_{\alpha}^{\mathcal{M}}}{d s_{\alpha 0}}\left\langle s_{\alpha^{1} / 2}^{\mathcal{M}}\right\rangle_{\mathcal{M}}- \\
\sqrt{\varepsilon} \frac{d \lambda_{\alpha}^{\mathcal{M}}}{d s_{\alpha 0}}\left\langle s_{\alpha 1 / 2}^{\mathcal{M}}\right\rangle_{\mathcal{M}}=\lambda_{\alpha}^{\mathcal{M}}\left(s_{\alpha 0}+\sqrt{\varepsilon}\left\langle s_{\alpha 1 / 2}^{\mathcal{M}}\right\rangle_{\mathcal{M}}\right)+ \\
\mathcal{O}(\sqrt{\varepsilon})=\lambda_{\alpha}^{\mathcal{M}}\left(\mathcal{S}_{\alpha}^{\mathcal{M}}\right)+\mathcal{O}(\sqrt{\varepsilon})
\end{gathered}
$$

Consequently, we deduce the following:

$$
\frac{\sqrt{\varepsilon}}{\lambda_{\alpha 0}^{\mathcal{M}}} \partial_{t} s_{\alpha 0}=\frac{\sqrt{\varepsilon} \partial_{t} \mathcal{S}_{\alpha}^{\mathcal{M}}+\mathcal{O}(\varepsilon)}{\lambda_{\alpha}^{\mathcal{M}}\left(\mathcal{S}_{\alpha}^{\mathcal{M}}\right)+\mathcal{O}(\sqrt{\varepsilon})}=\frac{\sqrt{\varepsilon} \partial_{t} \mathcal{S}_{\alpha}^{\mathcal{M}}}{\lambda_{\alpha}^{\mathcal{M}}\left(\mathcal{S}_{\alpha}^{\mathcal{M}}\right)}+\mathcal{O}(\varepsilon)
$$

and similarly for other terms.

Instead of two equations (49) for phase pressures, it is possible to replace one of them by the equation for the capillary pressure. Subtracting one equation (49) from another one, we deduce the relation between the averaged capillary pressures in the blocks and fractures:

$$
\begin{aligned}
& \mathcal{P}_{w}^{\mathcal{M}}=\mathcal{P}_{w}^{\mathcal{F}}+\tau_{w}^{\mathrm{com}} \partial_{t} \mathcal{P}_{w}^{\mathcal{M}}-\tau_{o}^{\mathrm{com}} \partial_{t}\left(\mathcal{P}_{w}^{\mathcal{M}}+\mathcal{P}_{c}^{\mathcal{M}}\right)+ \\
& \left(\frac{\tau_{w}^{\mathrm{com}}}{C_{w}^{\mathcal{M}} \mathcal{S}_{w}^{\mathcal{M}}}+\frac{\tau_{o}^{\mathrm{com}}}{C_{o}^{\mathcal{M}} \mathcal{S}_{o}^{\mathcal{M}}}\right) \partial_{t} \mathcal{S}_{w}^{\mathcal{M}}= \\
& \mathcal{P}_{w}^{\mathcal{F}}+\left(\frac{\tau_{w}^{\mathrm{com}}}{C_{w}^{\mathcal{M}} \mathcal{S}_{w}^{\mathcal{M}}}+\frac{\tau_{o}^{\mathrm{com}}}{C_{o}^{\mathcal{M}} \mathcal{S}_{o}^{\mathcal{M}}}-\tau_{w}^{\mathrm{com}} \frac{d \mathcal{P}_{c}^{\mathcal{M}}}{d \mathcal{S}_{w}^{\mathcal{M}}}\right) \partial_{t} \mathcal{S}_{w}^{\mathcal{M}}+ \\
& \left(\tau_{w}^{\mathrm{com}}-\tau_{o}^{\mathrm{com}}\right) \partial_{t} \mathcal{P}_{w}^{\mathcal{M}}
\end{aligned}
$$

two tween the aved to tween the average capillary pressures $\mathcal{P}_{c}^{\mathcal{M}}$ and $\mathcal{P}_{c}^{\mathcal{J}}$ and
the original capillary pressure curves $p_{c}^{\mathcal{M}}$ and $p_{c}^{\mathcal{F}}$. This is done in the following way. Let us expand the expression for $p_{c}^{\mathcal{M}}$ and $p_{c}^{\mathcal{F}}$ in Taylor series:

$$
\begin{aligned}
& p_{c}^{\mathcal{F}}\left(\mathcal{S}_{w}^{\mathcal{F}}\right)=p_{c}^{\mathcal{F}}\left(s_{w 0}\right)+\sqrt{\varepsilon} \frac{d p_{c}^{\mathcal{F}}}{d s_{w 0}} s_{w 1 / 2}^{\mathcal{F}}+\mathcal{O}(\varepsilon), \\
& p_{c}^{\mathcal{M}}\left(\mathcal{S}_{w}^{\mathcal{M}}\right)=p_{c}^{\mathcal{M}}\left(s_{w 0}\right)+\sqrt{\varepsilon} \frac{d p_{c}^{\mathcal{M}}}{d s_{w 0}}\left\langle s_{w^{1} / 2}^{\mathcal{M}}\right\rangle_{\mathcal{M}}+\mathcal{O}(\varepsilon)
\end{aligned}
$$

Since all the functions on the right do not depend on $y$, then the averaging over the fractures or blocks does not change anything:

$$
\begin{aligned}
& \mathcal{P}_{c}^{\mathcal{F}}\left(\mathcal{S}_{w}^{\mathcal{F}}\right) \equiv\left\langle p_{c}^{\mathcal{F}}\left(\mathcal{S}_{w}^{\mathcal{F}}\right)\right\rangle_{\mathcal{F}}=p_{c}^{\mathcal{F}}\left(\mathcal{S}_{w}^{\mathcal{F}}\right)+\mathcal{O}(\varepsilon), \\
& \mathcal{P}_{c}^{\mathcal{M}}\left(\mathcal{S}_{w}^{\mathcal{M}}\right) \equiv\left\langle p_{c}^{\mathcal{M}}\left(\mathcal{S}_{w}^{\mathcal{M}}\right)\right\rangle_{\mathcal{M}}=p_{c}^{\mathcal{M}}\left(\mathcal{S}_{w}^{\mathcal{M}}\right)+\mathcal{O}(\varepsilon)
\end{aligned}
$$

which proves (27). 


\section{E. Fifth step: final form of Eqs. (28a)}

For the expressions in the right-hand side of the macro-508 scopic equations (28a), it is possible to obtain an explicit ${ }^{509}$ relation through the pressure difference, using (28b): $\quad{ }^{510}$

$$
C_{\alpha}^{\mathcal{M}} \mathcal{S}_{\alpha}^{\mathcal{M}} \partial_{t} \mathcal{P}_{\alpha}^{\mathcal{M}}=-\frac{C_{\alpha}^{\mathcal{M}} \mathcal{S}_{\alpha}^{\mathcal{M}}}{\tau_{\alpha}^{\text {com }}}\left(\mathcal{P}_{\alpha}^{\mathcal{M}}-\mathcal{P}_{\alpha}^{\mathcal{F}}\right)
$$

which gives a more traditional form to the terms of ex-512 change between blocks and fractures and leads to the 513 definite form (28a).

\section{PHYSICAL INTERPRETATION OF THE RESULTS OBTAINED}

\section{A. Particular case of compressible single-phase flow}

In the single-phase case, system (28) takes the form:

$$
\left\{\begin{array}{l}
\phi^{\mathcal{F}} C^{\mathcal{F}}(1-\theta) \partial_{t} \mathcal{P}^{\mathcal{F}}-\partial_{x i}\left(\mathbb{K}_{i k} \partial_{x i} \mathcal{P}^{\mathcal{F}}\right)=\xi\left(\mathcal{P}^{\mathcal{M}}-\mathcal{P}^{\mathcal{F}}\right)_{524}^{523} \\
\mathcal{P}^{\mathcal{M}}=\mathcal{P}^{\mathcal{F}}-\tau^{\mathrm{com}} \partial_{t} \mathcal{P}^{\mathcal{M}}
\end{array}\right.
$$

where $\xi \equiv \frac{\theta}{\sqrt{\varepsilon}\langle\varphi\rangle_{\mathcal{M}}}, \tau^{\text {com }}=\sqrt{\varepsilon}\langle\varphi\rangle_{\mathcal{M}} \phi^{\mathcal{M}} C^{\mathcal{M}}$, which ${ }_{522}$ is the well-known model of single-phase flow in double ${ }_{530}^{529}$ porosity medium with moderate contrast ${ }^{11}$.

\section{B. Particular case of an incompressible two-phase system}

In the incompressible case, system (28) takes the fol- ${ }_{532}$ lowing form:

$$
\begin{gathered}
\phi^{\mathcal{F}}(1-\theta) \partial_{t} \mathcal{S}_{\alpha}^{\mathcal{F}}=\partial_{x i}\left(\mathbb{K}_{i k} \lambda_{\alpha}^{\mathcal{F}} \partial_{x i} \mathcal{P}_{\alpha}^{\mathcal{F}}\right)+ \\
\xi_{\alpha}\left(\mathcal{P}_{\alpha}^{\mathcal{M}}-\mathcal{P}_{\alpha}^{\mathcal{F}}\right), \quad \alpha=w, o \\
\mathcal{P}_{w}^{\mathcal{M}}=\mathcal{P}_{w}^{\mathcal{F}}-\frac{\tau_{w}^{\text {com }}}{C_{w}^{\mathcal{M}} \mathcal{S}_{w}^{\mathcal{M}}} \partial_{t} \mathcal{S}_{w}^{\mathcal{M}} \\
p_{c}^{\mathcal{M}}\left(\mathcal{S}_{w}^{\mathcal{M}}\right)=p_{c}^{\mathcal{F}}+\frac{\tau^{\text {cap }}}{C_{o}^{\mathcal{M}}} \partial_{t} \mathcal{S}_{w}^{\mathcal{M}}
\end{gathered}
$$

where parameters $\frac{\tau_{w}^{\text {com }}}{C_{w}^{\mathcal{M}} \mathcal{S}_{w}^{\mathcal{M}}}$ and $\frac{\tau^{\text {cap }}}{C_{o}^{\mathcal{M}}}$ do not depend on the ${ }^{540}$ compressibility coefficients:

$$
\begin{aligned}
& \frac{\tau_{w}^{\text {com }}}{C_{w}^{\mathcal{M}} \mathcal{S}_{w}^{\mathcal{M}}}=\sqrt{\varepsilon}\langle\varphi\rangle_{\mathcal{M}} \frac{\phi^{\mathcal{M}}}{\lambda_{w}^{\mathcal{M}}}, \\
& \frac{\tau^{\text {cap }}}{C_{o}^{\mathcal{M}}}=\sqrt{\varepsilon}\langle\varphi\rangle_{\mathcal{M}} \phi^{\mathcal{M}} \frac{\left(\lambda_{w}^{\mathcal{M}}+\lambda_{o}^{\mathcal{M}}\right)}{\lambda_{w}^{\mathcal{M}}}
\end{aligned}
$$

As seen from two last equations in (58), only the latter ${ }_{549}$ is differential (with respect to $\mathcal{S}_{w}^{\mathcal{M}}$ ), while the former is algebraic with respect to $\mathcal{P}_{w}^{\mathcal{M}}$. This means that the delay effects, which are caused only by the capillarity in this case, concern the capillary pressure and saturation. The difference in phase pressures in blocks and fractures also exists, but only as a consequence of the link of phase pressures with capillary pressure.

\section{Capillary delay and delay caused by compressibility}

Comparison with the case of incompressible fluids (58) and single-phase flow (57) enables us to better understand the essence of the obtained model (28) and the role of compressibility in two-phase systems. The nonequilibrium behavior of system (28) is determined by the subsystem of two ordinary differential equations (28b) and (28d) for saturation and pressure. This means that the pressure and the saturation of water in blocks are delayed with respect to those in fractures. Such a delay is caused by the following mechanisms on the microscale.

- Non-equilibrium capillary redistribution of phases between the blocks and fractures, caused by the fact that the average saturation in blocks changes more slowly than in fractures, which violates the equality of average capillary pressures (the condition of capillary equilibrium). As a result, a difference in the average capillary pressures arises, which depends on the rate of variation of the saturation in the blocks. In equation (28d), this process is described by the first term:

$$
p_{c}^{\mathcal{M}}-p_{c}^{\mathcal{F}}=\frac{\tau^{\text {cap }}}{C_{o}^{\mathcal{M}}} \partial_{t} \mathcal{S}_{w}^{\mathcal{M}}
$$

The difference in capillary pressures automatically causes a difference in phase pressures, which is expressed by the first term in (28b):

$$
\mathcal{P}_{w}^{\mathcal{M}}-\mathcal{P}_{w}^{\mathcal{F}}=\frac{\tau_{w}^{\text {com }}}{C_{w}^{\mathcal{M}} \mathcal{S}_{w}^{\mathcal{M}}} \partial_{t} \mathcal{S}_{w}^{\mathcal{M}}
$$

In a medium with strong contrast in permeabilisity, such a process would lead to long memory, but in the case of moderate contrast, we obtain the short memory described by the kinetic relationship (28d). This process does not depend on the compressibility of phases and rocks and is the same as in the incompressible case (58).

- Nonuniform pressure redistribution between the blocks and fractures. The compressibility of the system determines the appearance of pressure waves, whose rate of propagation is equal to $K /(\mu C \phi)$. Thus, it is lower in low permeable blocks than in fractures. This leads to a delay in the behavior of pressures, which is described by the second term in equation $(28 \mathrm{~b})$ :

$$
\mathcal{P}_{w}^{\mathcal{M}}-\mathcal{P}_{w}^{\mathcal{F}}=-\tau_{w}^{\text {com }} \partial_{t} \mathcal{P}_{w}^{\mathcal{M}}
$$


This process does not depend on the two-phase na-595 ture of the system and is the same as in the singlephase case, (57).

- Non-equilibrium asymmetric extrusion of the phases (peristalsis) due to their expansion and compaction of pores. Compressibility leads to expansion of liquids and compaction of pores under the weight of overlying rocks, which leads to the extrusion of both phases from the pores. This effect is similar to peristalsis, when a fluid in a chan- ${ }^{590}$ nel is driven by the deformation of the channel walls. If such extrusion is symmetrical for both ${ }^{597}$ phases, they are extruded as a whole, which does ${ }^{59}$ not change their volume fraction. In contrast, an599 asymmetrical extrusion leads to a redistribution of 600 phases in space, which affects their saturation. In601 blocks, such a movement caused by extrusion is de-602 layed, which involves an additional non-equilibrium603 in the saturation behavior, and enhances the cap-604 illary disequilibrium. This leads to an additional605 difference in capillary pressures, which is reflected600 by the third term in (28d):

$$
p_{c}^{\mathcal{M}}-p_{c}^{\mathcal{F}}=\left(\tau_{w}^{\mathrm{com}}-\tau_{o}^{\mathrm{com}}\right) \partial_{t} \mathcal{P}_{w}^{\mathcal{M}}
$$

As seen, this effect is really zeroed if the extrusion ${ }^{610}$ of the phases is symmetrical, that is if $\tau_{w}^{\mathrm{com}}-\tau_{o}^{\mathrm{com}}{ }_{{ }_{612}}$

- The nonlinear component of extrusion, which is ${ }^{613}$ also caused by the imposition of the capillarity, ${ }^{614}$ compressibility and nonlinearity of the flow equa-615 tions. This effect is described by the second term610 in $(28 \mathrm{~d})$ :

$$
p_{c}^{\mathcal{M}}-p_{c}^{\mathcal{F}}=\frac{\tau^{\mathrm{cc}}}{C_{o}^{\mathcal{M}}} \partial_{t} \mathcal{S}_{w}^{\mathcal{M}}
$$

\section{QUANTITATIVE ANALYSIS}

To analyse the role of various memory effects, we will consider two examples of the application of this model to an underground reservoir of oil or gas in an aquifer:

- Case I: monotonic depletion of an oil reservoir;

- Case II: oscillatory functioning of an underground gas storage in an aquifer.

In both cases we assume that the saturation and pres- ${ }^{625}$ sure in fractures are constant in space but vary in time ${ }^{626}$ as given functions, whose behaviour reflects the physical ${ }^{628}$ process we are analyzing. For instance, for the depletion ${ }^{629}$ process, the fracture pressure and saturation are mono-630 tonic functions of time, whilst for the gas storage they ${ }^{631}$ are oscillatory in time. Then it is sufficient to solve only ${ }^{632}$ the system of two differential equations (28b) and (28d), ${ }^{633}$ which can be presented in the following form:

$$
\left\{\begin{array}{l}
a_{11} \partial_{t} \mathcal{S}^{\mathcal{M}}+a_{12} \partial_{t} \mathcal{P}^{\mathcal{M}}=-\left(\mathcal{P}^{\mathcal{M}}-\mathcal{P}^{\mathcal{F}}\right) \\
a_{21} \partial_{t} \mathcal{S}^{\mathcal{M}}+a_{22} \partial_{t} \mathcal{P}^{\mathcal{M}}=p_{c}^{\mathcal{M}}-p_{c}^{\mathcal{F}}
\end{array}\right.
$$

where $\mathcal{S} \equiv \mathcal{S}_{w}, \mathcal{P} \equiv \mathcal{P}_{w}$, and

$$
\begin{aligned}
& a_{11} \equiv \frac{\tau_{w}^{\mathrm{com}}}{C_{w}^{\mathcal{M}} \mathcal{S}^{\mathcal{M}}}, \quad a_{12} \equiv \tau_{w}^{\mathrm{com}} \\
& a_{21} \equiv \frac{\left(\tau^{\mathrm{cap}}+\tau^{\mathrm{cc}}\right)}{C_{o}^{\mathcal{M}}}, \quad a_{22} \equiv \tau_{w}^{\mathrm{com}}-\tau_{o}^{\mathrm{com}}
\end{aligned}
$$

\section{A. Parameters of natural systems}

All the variables in the system (28) are assumed to be dimensionless.

The dimensionless value of compressibility parameter corresponds to $C_{w} p^{0}$ where $p^{0}$ is the initial pressure ((19)). In natural underground reservoirs, $p^{0} \sim 20-50$ $\mathrm{MPa}$. The compressibility of water and consolidated rocks (silicates) is of order $10^{-9}-10^{-8} \mathrm{~Pa}^{-1}$, which corresponds to dimensionless value of $C_{w} \sim 0.02-0.5$. The compressibility of oil and soft rocks is higher: $10^{-8}-10^{-7} \mathrm{~Pa}^{-1}$, so that the dimensionless compressibility is $C_{o} \sim 0.2-5$.

The exponential law of compressibility (1) is applied to liquids and solids. But it can also be used for highly compressed gas, whose properties are close to those of liquid. The compressibility of such a dense gas may be much higher than any liquid, so that the dimensionless parameter $C$ is of order $2-50$.

Thus, we conclude that:

- $C_{\alpha} \sim 0.03$ : low compressibility, $10^{-9} \mathrm{~Pa}^{-1}$;

- $C_{\alpha} \sim 0.3$ : moderate compressibility, $10^{-8} \mathrm{~Pa}^{-1}$;

- $C_{\alpha} \sim 3$ : high compressibility, $10^{-7} \mathrm{~Pa}^{-1}$;

- $C_{\alpha} \sim 30$ : very high compressibility (of dense gases), $10^{-6} \mathrm{~Pa}^{-1}$.

Other parameters are: $K^{\mathcal{F}}=1, K^{\mathcal{M}}=1, \varepsilon=0.2$, $\phi^{\mathcal{F}}=0.2, \phi^{\mathcal{M}}=0.2, \theta=0.8, \mu_{w} / \mu_{o}=0.5$. We use the following analytical curves of relative permeability and capillary pressure:

$$
\begin{aligned}
& k_{w}(s)=s^{2}, \quad k_{o}(s)=(1-s)^{2} \\
& p_{c}^{\mathcal{F}}(s)=\gamma \sqrt{-\ln s}, \quad p_{c}^{\mathcal{M}}(s)=\frac{\gamma}{\eta} \sqrt{-\ln s}
\end{aligned}
$$

where $\gamma=0.1$ and $\eta=0.65$. These functions are shown in Fig. 2. For the sake of simplicity, the relative permeabilities are assumed to be identical in blocks and fractures.

The cell problem (33) represents a Dirichlet problem for Poisson's equation. It was solved numerically in a square block by the finite element method using the Matlab PDE Toolbox, which is the solver of partial differential equations integrated into the package Matlab. The domain was discretized by triangular elements. The basic functions were linear. The solution has the form of a convex surface having the maximum at the block centre and zero at the block boundary. Its average value is $\langle\varphi\rangle_{\mathcal{M}}=0.025$. 


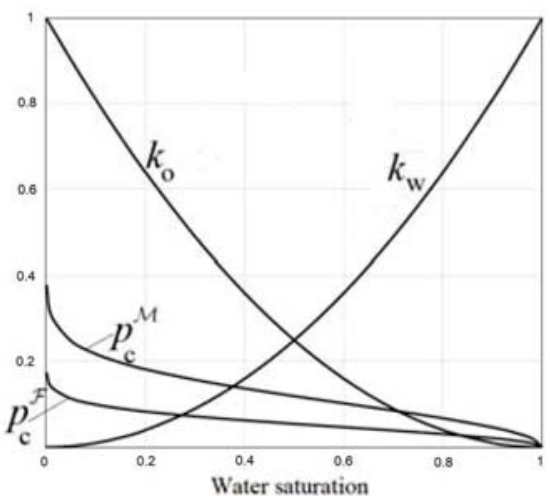

FIG. 2. Original curves of relative permeability and capillary pressure

\section{B. Monotonic depletion of an oil reservoir}

Let the reservoir contain initially oil and water. The initial pressure is uniform in the entire reservoir, while the saturations are different in blocks and fractures, being controlled by the capillary equilibrium:

$$
\mathcal{P}_{w}^{\mathcal{F}}=\mathcal{P}_{w}^{\mathcal{M}}=1, \quad \mathcal{S}_{w}^{\mathcal{F}}=0.2, \quad \text { at } \quad t=0
$$

The production of oil leads to the decrease in reservoir pressure and to the invasion of the aquifer water, which leads, in turn, to the increase in water saturation. This can be expressed in terms of the following behaviour of the pressure and saturation in fractures:

$$
\mathcal{P}_{w}^{\mathcal{F}}=0.7-q t, \quad \mathcal{S}_{w}^{\mathcal{F}}=0.2+q t
$$

where $q$ is the depletion rate.

This means that $\mathcal{P}_{w}^{\mathcal{F}}$ instantaneously drops from 1 to 0.7 and then decreases linearly with time.

The behaviour of block pressure and saturation is shown in Fig. 3 for high compressibility: $C_{w}=C_{o}=3$.

A significant delay in pressure propagation is observed only for highly compressible systems $\left(C_{w} \gg 1\right)$.

The saturation field is much more affected by the compressibility effects than pressure.

\section{Oscillatory regimes of injection-production}

Let the pressure and saturation in fractures oscillate in time, which corresponds in practice to functioning of an underground storage of gas in an aquifer. A half of year the gas in injected (the pressure increases and the water saturation decreases). For another half year, the gas is withdrawn, so that the pressure decreases and the water saturation increases:

$$
\mathcal{P}^{\mathcal{F}}=1+A \cos (\nu t), \quad \mathcal{S}^{\mathcal{F}}=0.2+A \sin (\nu t)
$$

where $A$ and $\nu$ are the amplitude and the period of oscillations.

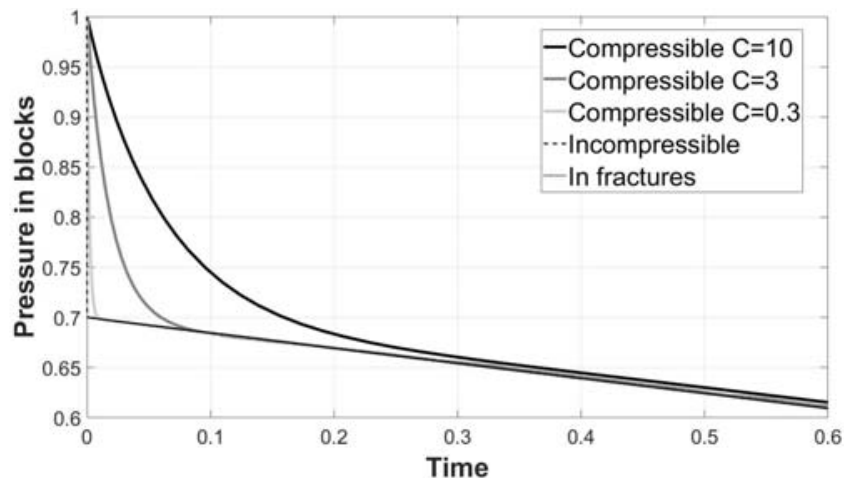

(a)

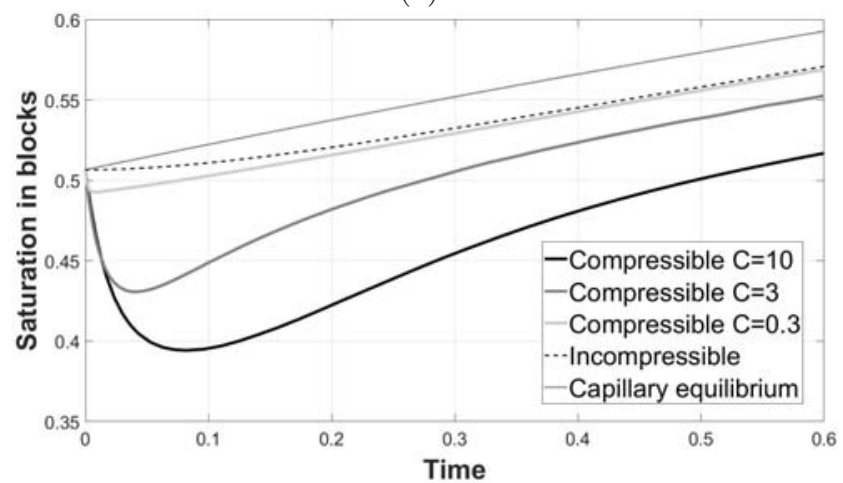

(b)

FIG. 3. Variation of pressure (a) and water saturation (b) in blocks, for various $C_{w}^{\mathcal{M}}=C_{o}^{\mathcal{M}}=0.3,3,10$

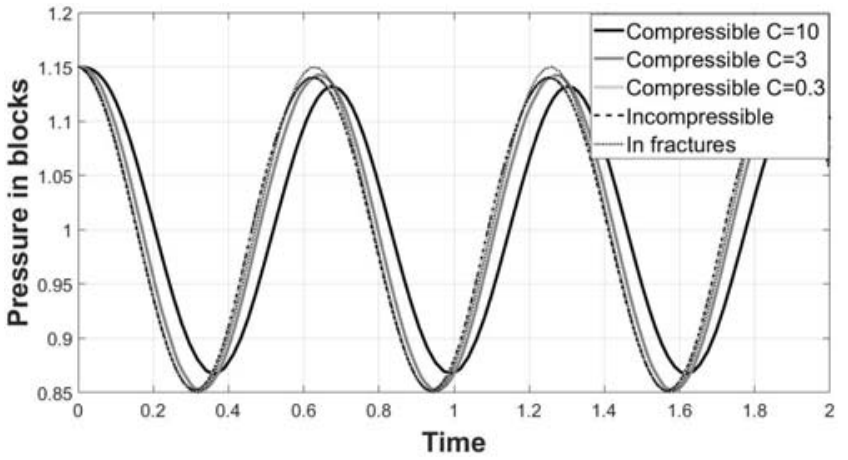

(a)

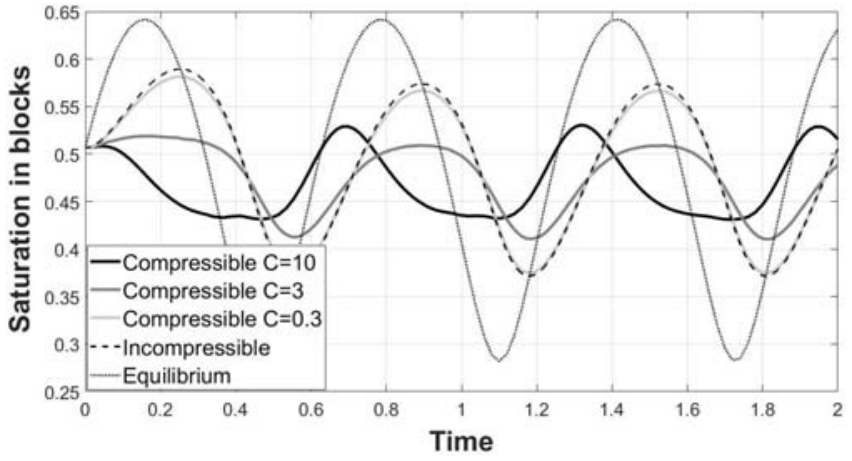

(b)

FIG. 4. Variation of pressure (a) and saturation (b) in blocks, for various $C_{w}^{\mathcal{M}}=C_{o}^{\mathcal{M}}=0.3,3,10$ 
The behaviour of pressure and saturation is shown in Fig. 4, for high compressibility $(C \sim 3)$.

As in the previous case, the pressure delay is observed only for very high compressibility of fluids or rocks. In contrast, the delay in the saturation is significant even for moderate values of compressibility $C_{w}, C_{o} \sim 1-3$. In the case of high compressibility, the saturation in blocks is totally different from the incompressible case.

\section{Impact of asymmetrical compressibility of phases}

If the compressibility of two phases is very different, it has a significant impact on saturation, even for moderate compressibility. This impact is even greater than that of a strong but identical compressibility for the two phases. This is clearly seen in Fig. 5, which compares ${ }^{708}$ two cases: the high compressibility but identical for both ${ }^{709}$ phases (the grey curve, $C_{w}=C_{o}=3$ ), and the case of different compressibility (the black curve, $C_{w}=0.3$ and $\left.C_{o}=3\right)$.

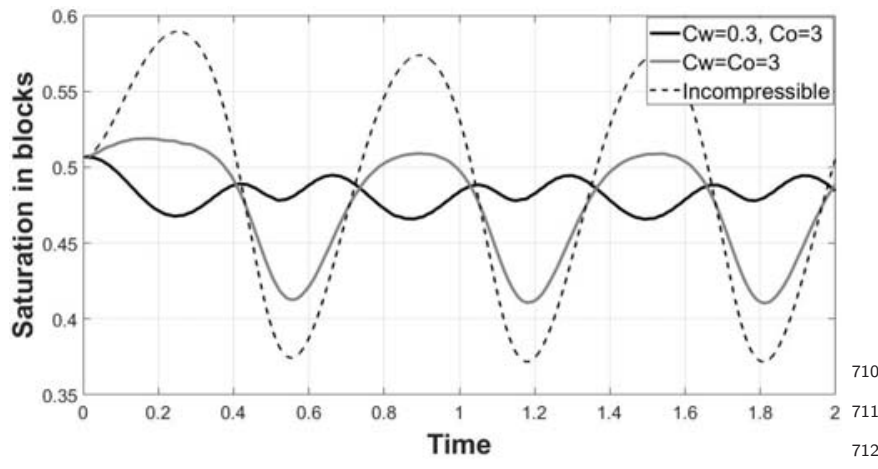

. Variation of the saturation in blocks ir asymetrical compressibility, for $C^{\mathcal{M}}=0.3$ and $C^{\mathcal{M}}=3$

If the compressibility is asymmetrical and high, then the saturation in blocks changes even qualitatively the ${ }_{718}$ behaviour with respect to the incompressible case. $\operatorname{In}_{719}$ Fig. 6 the black curve (high compressibility of both ${ }_{720}$ phases) has not only much higher amplitude but also ${ }_{721}$ the inverse maximums/minimus comparing to the incom-72z pressible case (the dashed curve) or to the case of $a_{724}$ identical compressibility (the grey curve).

In all the situations, one sees that the delay effects concern much more the saturation, than the pressure. A moderate and even low but asymmetrical compressibility ${ }_{727}$ has significant impact on the saturation.

\section{E. Simplification of the macroscopic model}

Given the results of simulations, we can suggest a sim-732 plified approximate macroscopic model, in which we ne-733 glect the delay in the behaviour of pressure. Then the ${ }_{734}$ version of the model (28), in which only the saturation 735

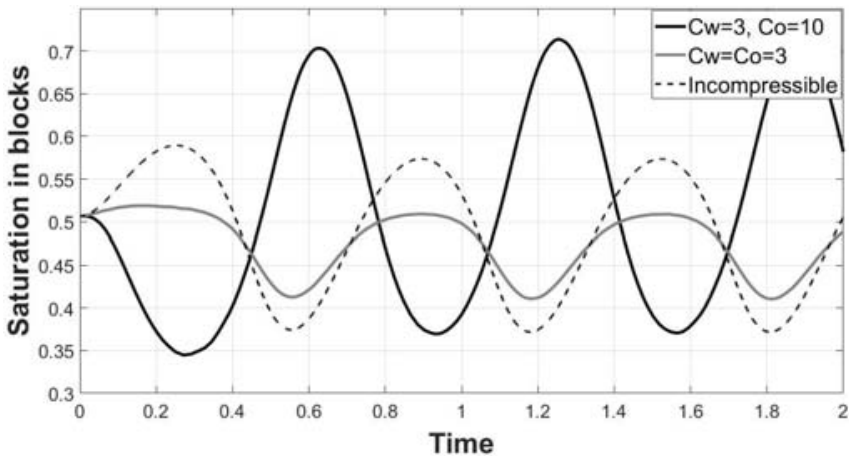

FIG. 6. Variation of the saturation in blocks in the case of asymmetrical compressibility, for $C_{w}^{\mathcal{M}}=3$ and $C_{o}^{\mathcal{M}}=10$

is in disequilibrium (but not the pressure) takes the following form:

$$
\begin{aligned}
& \phi^{\mathcal{F}}(1-\theta) \partial_{t} \mathcal{S}_{\alpha}^{\mathcal{F}}+\phi^{\mathcal{F}} C_{\alpha}^{\mathcal{F}}(1-\theta) \mathcal{S}_{\alpha}^{\mathcal{F}} \partial_{t} \mathcal{P}_{\alpha}^{\mathcal{F}}= \\
& \partial_{x i}\left(\mathbb{K}_{i k} \lambda_{\alpha}^{\mathcal{F}} \partial_{x i} \mathcal{P}_{\alpha}^{\mathcal{F}}\right), \quad \alpha=w, o \\
& \mathcal{P}_{w}^{\mathcal{M}}=\mathcal{P}_{w}^{\mathcal{F}}, \\
& \frac{\tau^{\mathrm{cap}}+\tau^{\mathrm{cc}}}{C_{o}^{\mathcal{M}}} \partial_{t} \mathcal{S}_{w}^{\mathcal{M}}=p_{c}^{\mathcal{M}}\left(\mathcal{S}_{w}^{\mathcal{M}}\right)-p_{c}^{\mathcal{F}}- \\
& \quad\left(\tau_{w}^{\mathrm{com}}-\tau_{o}^{\mathrm{com}}\right) \partial_{t} \mathcal{P}_{w}^{\mathcal{M}}
\end{aligned}
$$

Contrarily to the exact model (28), the first two equations (70a) do not contain anymore the exchange term, due to a fast equalization of pressure in space. Moreover, two kinetic differential equations (28b) and (28d) transform into a single ordinary differential equation (70d) with respect to the saturation $\mathcal{S}_{w}^{\mathcal{M}}$.

Equation (70d) can be considered as the generalization to compressible systems of the capillary non-equilibrium equation obtained in ${ }^{13}$.

This simplified model is valid for $C_{w}, C_{o}<3-4$. These dimensionless values correspond to the compressibility of $10^{-7} \mathrm{~Pa}^{-1}$, which is a highly compressible system. Fig. 7 illustrates this.

This is however not the case of very high compressibility, as illustrated in Fig. 8.

\section{CONCLUSION}

First of all, we note that the averaged model (28) is completely homogenized, despite the presence of the nonlinearity and the memory. This means that it does not contain microscopic variables, and the cell problem does not contain macroscopic variables, so that it is solved only once.

This was possible to do due to the method of splitting nonlocality and nonlinearity, proposed earlier ${ }^{13}$, which 


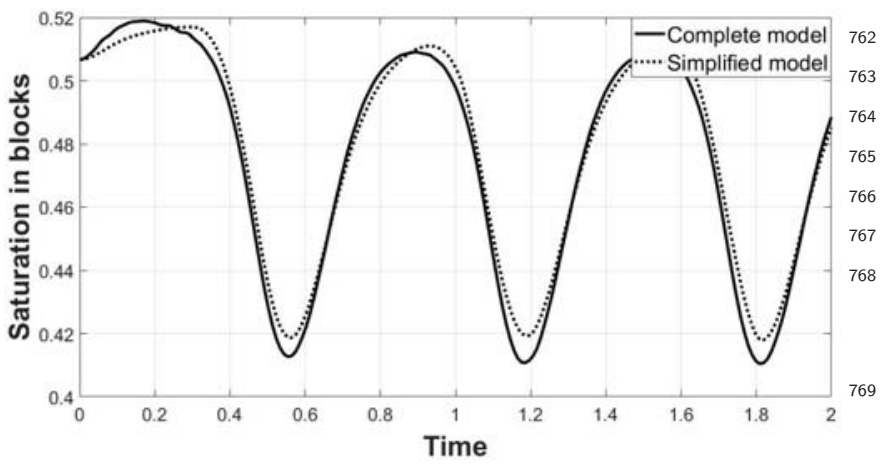

FIG. 7. Comparison of the simplified model (the dotted ${ }^{771}$ curve) and the exact model (the solid curve) for $C_{w}=C_{o}=3772$

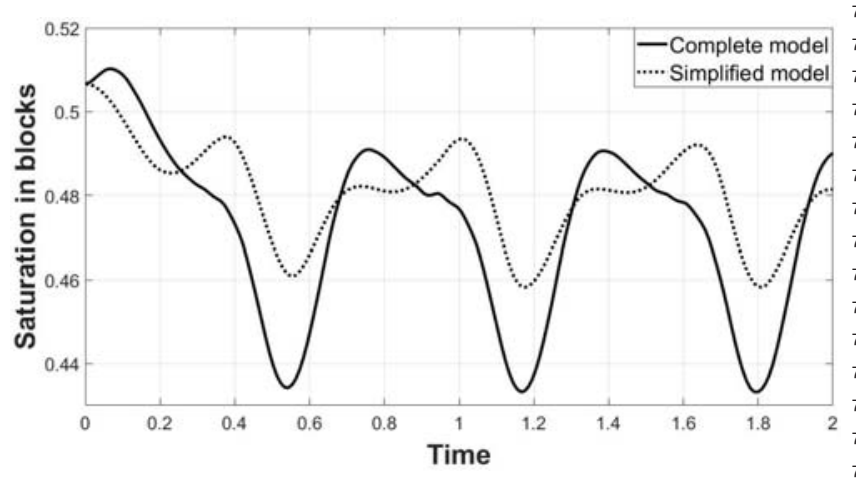
curve) and the exact model (the solid curve) for a highly com-790 pressible system: $C_{w}=C_{o}=5$ 776 777 778 781 782 (1)

\section{,}

showed once again its effectiveness. Averaging by the ${ }_{795}^{794}$ variational method (which remains non-popular yet in $n_{796}$ mechanics of porous media), is also highly efficient, since ${ }^{797}$ it allows minimizing the calculations.

Secondly, the model predicts and describes several ${ }_{800}^{799}$ mechanisms causing the delay or memory effects. Along ${ }_{801}^{800}$ with the pure capillary delay, typical to two-phase sys-802 tems, and the delay of pressure wave propagation, typi-803 cal for compressible systems, two additional cross-effects ${ }^{804}$ arise, such as peristalsis (or phase extrusion) and non ${ }_{806}^{805}$ linear peristalsis, which, in turn, depend non-trivially on ${ }_{807}^{806}$ the asymmetry or symmetry of the compression with re-808 spect to two phases.

The practical application of this model is necessary ${ }_{811}^{810}$ in cases of strongly non-stationary processes, when com- ${ }^{811}$ pressibility plays a significant role in the propagation of ${ }_{813}$ perturbations.

Various memory effects are particularly pronounced in ${ }^{815}$ processes with a variable direction of evolution. For $\mathrm{ex}^{-116}$ ample, the gas injection-production in underground gas $_{818}^{817}$ storage. The accumulation of a history of pressure and $\mathrm{d}_{819}$ saturation oscillations in such processes can lead to a820 strong delay and a complete discrepancy in the quali-- ${ }^{821}$ tative behavior of the process compared to the process $^{822}$ without memory.

We considered only the case of the exponential com-825 pressibility law, which is applicable for liquids and solids. The applicability of this model for a gas-liquid system is acceptable only for high pressures, or for highly compressed gas, whose properties are close to a liquid.

The delay caused by the compressibility affects the saturation (through the asymmetrical peristalsis) even at moderate values of compressibility parameter.

\section{ACKNOWLEDGMENT}

The author is grateful to prof. Alain Bourgeat from Université de Lyon, for fruitful discussions on homogenization of differential equations.

${ }^{1}$ A. Ainouz. Homogenized double porosity models for poro-elastic media with interfacial flow barrier. Mathematica Bohemica, 136(4), 357 - 365 (2011).

${ }^{2}$ Ait Mahiout L., Amaziane B., Mokrane A., and Pankratov L. Homogenization of immiscible compressible twophase flow in double porosity media. Electronic Journal of Differential Equations, 2016:52, 1 - 28 (2016).

${ }^{3} \mathrm{G}$. Allaire. Homogenization and two-scale convergence. SIAM J. Math. Anal., 28, 1482 - 1518 (1992).

${ }^{4}$ B. Amaziane, M. Jurak, L. Pankratov, A. Vrbaski, Some remarks on the homogenization of immiscible incompressible two-phase flow in double porosity media. Discrete and Continuous Dynamical Systems - Series B, 2018, 23(2), 629 - 665 (2018).

${ }^{8}$ B. Amaziane B., and L. Pankratov. Homogenization of a model for water-gas flow through double-porosity media. Mathematical Methods in the Applied Sciences, 39, 425 - 451 (2016).

${ }^{6}$ B. Amaziane, L. Pankratov, M. Jurak, A. Vrbaski. A fully homogenized model for incompressible two-phase flow in double porosity media. Applicable analysis, April, (2015)

${ }^{7}$ B. Amaziane, J.P. Milisic, M. Panfilov, and L. Pankratov. Generalized nonequilibrium capillary relations for two-phase flow through heterogeneous media. Phys. Review E, 85, 016304 (2012).

${ }^{8}$ B. Amaziane, M. Panfilov, and L. Pankratov. Homogenized model of two-phase flow with local nonequilibrium in double porosity media. Advances in Math. Physics, Article ID 3058710 (2016), http://dx.doi.org/10.1155/2016/3058710.

${ }^{9} \mathrm{~T}$. Arbogast, J. Douglas, and U. Hornung. Derivation of the double porosity model of single phase flow via homogenization theory. SIAM J. Appl. Math., 21, 823 (1990).

${ }^{10} \mathrm{~T}$. Arbogast. A simplified dualporosity model for twophase flow. In: Computational Methods in Water Resources IX, Vol. 2: Mathematical Modeling in Water Resources. Eds T.F. Russell, R.E. Ewing, C.A. Brebbia, W.G. Gray, and G.F. Pindar, (Computational Mechanics Publications, Southampton, 1992).

${ }^{11} \mathrm{G}$. Barenblatt, Y. Zheltov, and I. Kochina. On basic concepts of the theory of homogeneousfluids seepage in fractured rocks. Prikl. Mat. Mekh., 24852864 (1960) (in Russian).

${ }^{12}$ A. Bourgeat, S. Luckhaus, and A. Mikelić. Convergence of the homogenization process for a double-porosity model of immiscible two-phase flow. SIAM J. Math. Anal., 27(6), 1520 - 1543 (1996).

${ }^{13}$ A. Bourgeat, and M. Panfilov. Effective two-phase flow through highly heterogeneous porous media: Capillary nonequilibrium effects. Computational Geosciences, 2(3), 191 - 215 (1998).

${ }^{14} \mathrm{~B}$. Dastvareh, and J. Azaiez. Instabilities of nanofluid flow displacements in porous media. Phys. Fluids, 29(4), 044101 (2017).

${ }^{15} \mathrm{M}$. Hussein. Multiphase flow simulations in heterogeneous fractured media through hybrid grid method AIP Conference Proceedings 1558, 2048 (2013).

${ }^{16}$ I. Jafari, M. Masihi, and M. Nasiri Zarandi. Numerical simulation of counter-current spontaneous imbibition in water-wet fractured porous media: Influences of water injection velocity, frac- 
ture aperture, and grains geometry. Phys. Fluids, 29(11), 113305846 (2017).

${ }^{17}$ I. Jafari, M. Masihi, and M. Nasiri Zarandi. Experimental study 848 on imbibition displacement mechanisms of two-phase fluid us-849 ing micromodel: Fracture network, distribution of pore size, and850 matrix construction. Phys. Fluids, 29(11), 122004 (2017).

${ }^{18}$ Y. Khoshkalam, M. Khosravi, and B. Rostami. Visual investi-852 gation of viscous cross-flow during foam injection in a matrix-853 fracture system Phys. Fluids, 31, 023102 (2019).

${ }^{19}$ A. Konyukhov, L. Pankratov, A. Voloshin. The homogenized 855 Kondaurov type non-equilibrium model of twophase flow in mul-856 tiscale non-homogeneous media. Physica Scripta, 94 054002, 1 -857 15 (2019).

${ }^{20}$ H. Li, H. Guo, Z. Yang, H. Ren, L. Meng, H. Lu, H. Xu, Y. Sun, 859 T. Gao, and H. Zhang. Evaluation of oil production potential in860 fractured porous media. Phys. Fluids, 31, 052104 (2019).

${ }^{21}$ M. Panfilov, R. Marmier, and L. Jeannin. Averaged model of 862 a cross hydrodynamic-mechanic process in a double porosity 863 medium. Comptes Rendus Acad. Sci. Paris, Sér. IIb, Mécanique, 864 334, 190195 (2006).
${ }^{22}$ M. Panfilov, Macroscale models of flow through highly heterogeneous porous media. Kluwer Academic Publishers, Dordrecht, Boston, London, 2000.

${ }^{23}$ A. A. Pyatkov, V. P. Kosyakov, S. P. Rodionov, and A. Y. Botalov. Numerical research of two-phase flow in fractured-porous media based on discrete fracture fetwork model. AIP Conference Proceedings 1939, 020039 (2018).

${ }^{24}$ M. R. Rokhforouz, and H. A. Akhlaghi Amiri. Phase-field simulation of counter-current spontaneous imbibition in a fractured heterogeneous porous medium. Phys. Fluids, 29, 062104 (2017).

${ }^{25}$ B. Saedi, S. Ayatollahi, and M. Masihi. Free fall and controlled gravity drainage processes in fractured porous media: Laboratory and modelling investigation. Can. J. Chem. Eng. 93, 2286 (2015).

${ }^{26}$ D. Spiridonov, and M. Vasilyeva. Multiscale model reduction of the flow problem in fractured porous media using mixed generalized multiscale finite element method. AIP Conference Proceedings 2025, 100008 (2018).

${ }^{27}$ C. C. Yao, and P. Y. Yan. A diffuse interface approach to injection-driven flow of different miscibility in heterogeneous porous media. Phys. Fluids, 27(8), 083101 (2015).

${ }^{28}$ L. M. Yeh. Homogenization of two-phase flow in fractured media. Math. Models Methods Appl. Sci., 16, 1627 - 1651 (2006). 